\title{
Oxygen and carbon isotope signatures in late Neogene horse teeth from Spain and application as temperature and seasonality proxies
}

\author{
J.A. van Dam ${ }^{\text {a,b,*, G.J. Reichart }}{ }^{\mathrm{a}}$ \\ ${ }^{a}$ Faculty of Geosciences, Department of Earth Sciences, Utrecht University, Budapestlaan 4, 3584 CD, Utrecht, The Netherlands \\ ${ }^{\mathrm{b}}$ Faculty of Earth- and Lifesciences, Department of Paleoclimatology and Geomorphology, De Boelelaan 1085, 1081 HV, Amsterdam, The Netherlands
}

\section{A R T I C L E I N F O}

\section{Article history:}

Received 15 January 2008

Received in revised form 19 December 2008

Accepted 24 December 2008

\section{Keywords:}

Stable isotopes

Mammals

Neogene

Temperature

Seasonality

Spain

\begin{abstract}
A B S T R A C T
Here we describe a data set of 249 stable $O$ and C isotope compositions from fossil horse teeth from the Upper Neogene of Central Spain and discuss their potential as environmental proxies. It has been shown before that horse tooth enamel $\delta^{18} \mathrm{O}$ is a good proxy for local meteoric water $\delta^{18} \mathrm{O}_{\mathrm{H} 2 \mathrm{O}}$, which, in turn, bears a distinct relation to temperature during precipitation formation at mid to high latitudes. We discuss the reconstructed $\delta^{18} \mathrm{O}_{\mathrm{H} 2 \mathrm{O}}$ values, and apply modern $\delta^{18} \mathrm{O}_{\mathrm{H} 2 \mathrm{O}}$ - temperature equations for the Iberian Peninsula to these values. Results for the Early Pliocene suggest mean annual temperatures up to $2-3{ }^{\circ} \mathrm{C}$ higher than today, fitting pollen-based estimates fairly well. Temperatures $5{ }^{\circ} \mathrm{C}$ higher than today are predicted for the early Late Miocene (11-9 Ma). These estimations are feasible given the local occurrence of crocodilians and the presence of fruit-eating hominids in Iberia at that time. The estimated amount of cooling since then is similar to the estimated decrease in NW Europe based on plants. The stable carbon isotope record indicates the virtual absence of $\mathrm{C} 4$ plants, pointing to an environment consisting of C3 plants under slightly waterstressed conditions. The long-term $\delta^{13} \mathrm{C}$ trend follows the expected decrease in average global $\delta^{13} \mathrm{C}_{\mathrm{CO} 2}$ predicted from planktonic foraminifers.

Within-tooth variability is supposed to reflect seasonal change, but the amplitude is expected to have been significantly damped by internal time-averaging effects related to the mixing with atmospheric $\mathrm{O}_{2}$, reservoir dynamics, enamel maturation and sampling procedure. The exact degree of damping is difficult to estimate, but theoretical considerations suggest values between 50 and 75\%, depending on temporal resolution and element used $(\mathrm{C} / \mathrm{O})$. Reconstructed seasonal temperature ranges for Central Spain are equal or slightly less than the modern range. Within-tooth $\delta^{13} \mathrm{C}$ variation is more difficult to explain, as it can be attributed to multiple factors, such as intra-annual changes in $\delta^{13} \mathrm{C}_{\mathrm{CO} 2}$, micrometeorology, plant response and diet. The occurrence of both positive and negative intra-tooth $\delta^{18} \mathrm{O}-\delta^{13} \mathrm{C}$ correlations may be related to orbitallyforced variations in seasonality.
\end{abstract}

(C) 2009 Elsevier B.V. All rights reserved.

\section{Introduction}

The late Neogene represents an important period in Cenozoic climatic history because of the transition from unipolar (Antarctic) to bipolar glacial conditions. Significant global cooling took place, with mean annual temperatures dropping $\sim 7^{\circ} \mathrm{C}$ since 14 million years ago (Lear et al., 2000; Zachos et al., 2001). Spatial and seasonal aspects of this cooling episode are still poorly known, however. It is generally assumed that high-latitude and mid-continental regions underwent stronger cooling than tropical and marine environments. Also, the amount of cooling appeared to have differed per season, with most late Neogene cooling probably taking place during the cold season (Mosbrugger et al., 2005). Unfortunately, the number of well-documented and well-dated

\footnotetext{
* Corresponding author. Institut Català de Paleontologia, Universitat Autònoma de Barcelona, Bellaterra (08193), Barcelona, Spain. Tel.: +34935868343.

E-mail address: jan.vandam@icp.cat (J.A. van Dam).
}

Pre-Pleistocene regional temperature records is still low. Proxy records of changes in the seasonal cycle are even more rare.

Terrestrial temperature estimates are mostly based on paleobotanical data such as pollen or macrofloral remains (e.g. Wolfe, 1993; Fauquette et al., 1998; Utescher et al., 2000; Mosbrugger et al., 2005). However, well-represented fossil floras are relatively rare, especially in arid regions. Stable oxygen isotope compositions of fossil mammal teeth may provide an alternative source for temperature inferences, because these compositions allow the estimation of the composition in the precipitation, the formation of which is temperature-dependent. Particularly tooth enamel is very suitable for mammal isotopic studies, because it is extremely dense and resistant to diagenetic effects (Koch et al., 1997; Kohn and Cerling, 2002). In addition, fossil mammals have an important intrinsic stratigraphic value because of their common occurrence and rapid evolution.

Because mammals have a constant internal body temperature, the main determinant of their skeletal $\delta^{18} \mathrm{O}$ values is the $\delta^{18} \mathrm{O}$ of ingested water. The exact relation between the two quantities depends on the 


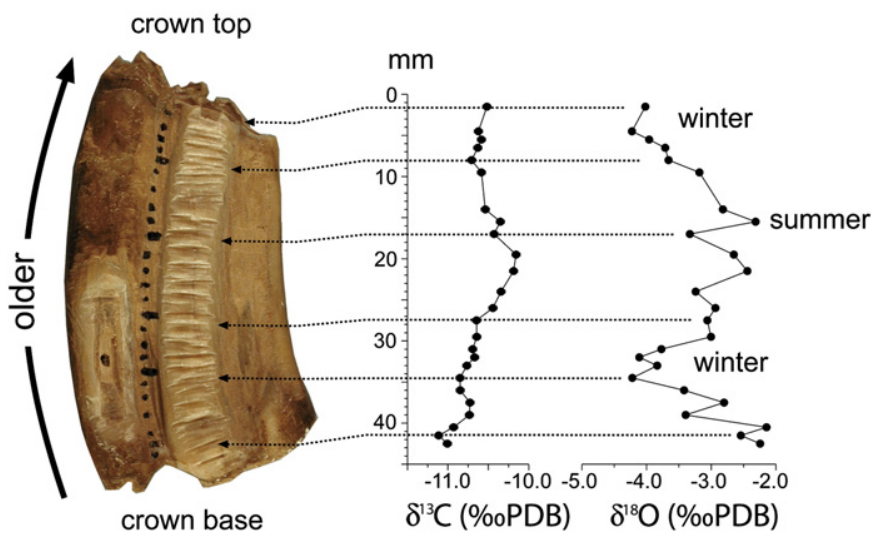

Fig. 1. Stable carbon and oxygen isotope profiles across a lower third molar (PM-212) of Hipparion primigenium from the locality Puente Minero, Teruel Basin. The tooth shows the positions of 25 sample furrows in the enamel. The left part of the tooth shows a spot where enamel is removed and dentine is exposed.

metabolic parameters of the studied taxon (Longinelli, 1984; Luz et al., 1984). Unless the animal obtains its water largely from plants, the $\delta^{18} \mathrm{O}$ of ingested water is usually very close to that of precipitation $\left(\delta^{18} \mathrm{O}_{\mathrm{H} 2 \mathrm{O}}\right)$ (Longinelli, 1984; Luz et al., 1984; Koch, 1998; Kohn and Cerling, 2002). The latter is related to temperature because of the preferential removal of ${ }^{18} \mathrm{O}$ from air masses during condensation when they cool (Dansgaard, 1964; Kohn and Welker, 2005). Because most air masses have their ultimate source in the tropics, a clear latitudinal poleward trend of decreasing $\delta^{18} \mathrm{O}_{\mathrm{H} 2 \mathrm{O}}$ in the precipitation can be observed. This latitudinal effect is modulated by the secondary effects of continentality, altitude and vertical convective transport, which all contribute to further preferential removal of ${ }^{18} \mathrm{O}$ (Dansgaard, 1964; Jouzel et al., 1987; Rozanski et al., 1992, 1993; Kendall and Coplen, 2001). A distinct $\delta^{18} \mathrm{O}_{\mathrm{H} 2 \mathrm{O}}$ - temperature relationship is absent at lower latitudes because latitudinal temperature differences are small and because strong convective rains occur. Instead, tropical $\delta^{18} \mathrm{O}_{\mathrm{H} 2 \mathrm{O}}$ primarily correlates with the amount of rainfall ("amount effect"). Also at higher latitudes, the amount effect may play a role during heavy rainfall events (Celle-Jeanton et al., 2001).

$\delta^{18} \mathrm{O}_{\mathrm{H} 2 \mathrm{O}}$ - temperature slopes at mid- and high latitudes are typically $0.5-0.8 \% /{ }^{\circ} \mathrm{C}$, although values up to $1.1 \% /{ }^{\circ} \mathrm{C}$ may be reached at the highest latitudes (Rozanski et al., 1992). There has been some discussion in the literature whether these spatial slopes can be used temporally as well. Both observations and models show that slopes are less when seasonal (monthly) data are used (around 0.3 for Europe, Rozanski et al., 1993), which could be due to the fact that within-year oceanic source temperatures do not change to the same degree as the final destination compositions on land. This effect does not play a role when two destination areas are compared (Rozanski et al., 1992, 1993; Cole et al., 1999; Jouzel et al., 2000). It has also been shown that discrepancies largely disappear when the actual temperatures during precipitation events are used instead of mean (e.g. monthly) temperatures (Kohn and Welker, 2005). The incorporation of such highresolution temporal data results in temporal slopes converging to the average mid-latitude spatial slope value of $0.55 \% /{ }^{\circ} \mathrm{C}$ (Fricke and O'Neil, 1999). In this paper we specifically focus on the Iberian Peninsula, deriving equations for predicting temperature from $\delta^{18} \mathrm{O}_{\mathrm{H} 2 \mathrm{O}}$ and discussing and applying these to the Late Neogene using $\delta^{18} \mathrm{O}$ from fossil horse tooth enamel.

Until now few temperature predictions on the basis of mammal tooth $\delta^{18} \mathrm{O}$ have been made. This is mainly due to the existence of various other factors affecting the $\delta^{18} \mathrm{O}$ in precipitation and drinking waters, resulting in large uncertainties associated with the predicting equations (Koch, 1998; Passey et al., 2002). Also, the number of stable isotope studies (such as on $\mathrm{O}, \mathrm{C}, \mathrm{Sr}$ ) that have been performed on European pre-Pleistocene Late Neogene mammal fossils is small to begin with. Late Pliocene horse $\delta^{18} \mathrm{O}$ data were published for Southern Spain by Sanchez Chillón (1993) and Sánchez-Chillón et al. (1994), who also published a relative temperature curve based on these data. Quade et al. (1994) published $\delta^{13} \mathrm{C}$ and $\delta^{18} \mathrm{O}$ measurements from large herbivore tooth enamel in Greece. In their global compilation of MioPliocene terrestrial $\delta^{13} \mathrm{C}$, Cerling et al. (1997) published a set of European equid data (mainly from France). More recently, Tütken et al. (2006) studied C, O and Sr isotopes from tooth enamel from the late Middle Miocene locality Steinheim (Germany), and used $\delta^{18} \mathrm{O}$ measurements to infer atmospheric temperatures. In this study, the authors combined isotopic measurements on mammal fossils with measurements on aquatic invertebrates, which allowed them estimate water temperatures as well. A similar approach was taken by Grimes et al. (2003) using Paleogene fossils. Furthermore, new Middle Miocene temperature estimates were published by Domingo et al. (2009) for the site of Somosaguas (Madrid Basin, Spain), based on stable $\mathrm{O}$ isotope compositions in the fossil enamel of various large mammal taxa including the horse Anchitherium.

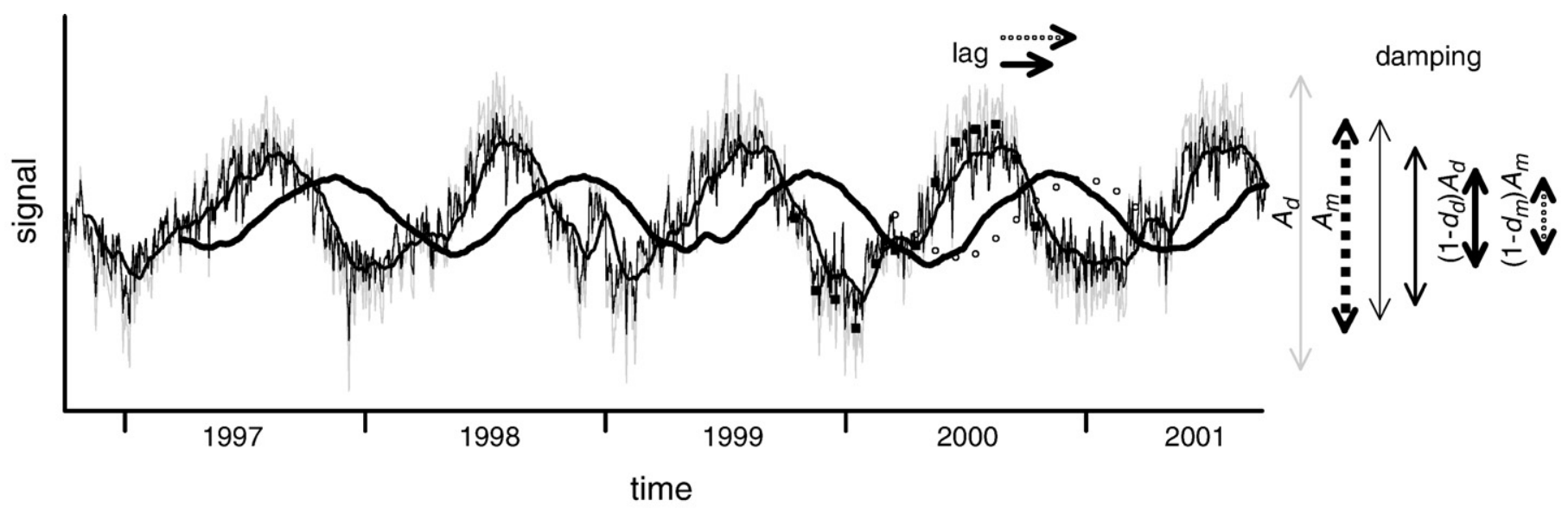

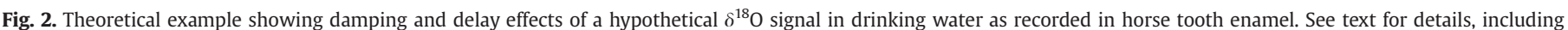

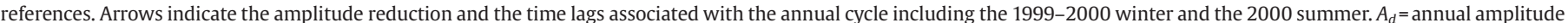

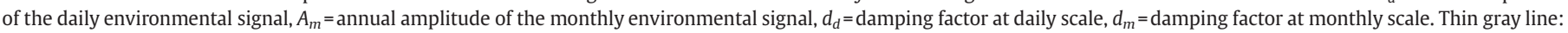

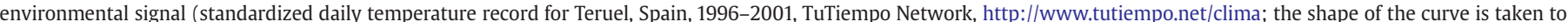

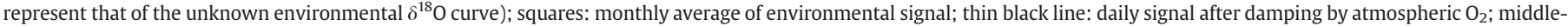

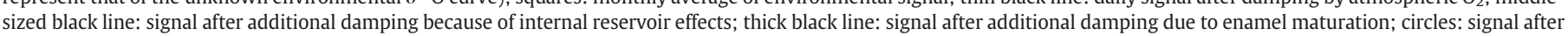

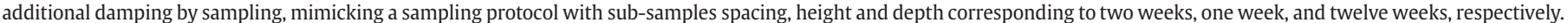


Table 1

$\delta^{18} \mathrm{O}$ and $\delta^{13} \mathrm{C}$ measurements on structural carbonate from horse tooth enamel from localities in east Central Spain

\begin{tabular}{|c|c|c|c|c|c|c|c|c|c|c|c|c|}
\hline Genus & Species & Locality & Locality code & Basin & Collection & Age (Ma) & Specimen & Element & $\mathrm{mm}$ from top & Laboratory & $\delta^{13} \mathrm{C}$ & $\delta^{18} \mathrm{O}$ \\
\hline Equus & stenonis & La Puebla de Valverde & PVAL & Teruel & MNCN & 2.1 & 1609 & M3 & 5 & Univ. Utrecht & -11.3 & -4.5 \\
\hline Equus & stenonis & La Puebla de Valverde & PVAL & Teruel & MNCN & 2.1 & 1609 & M3 & 9 & Univ. Utrecht & -11.4 & -4.3 \\
\hline Equus & stenonis & La Puebla de Valverde & PVAL & Teruel & MNCN & 2.1 & 1609 & M3 & 16 & Univ. Utrecht & -11.4 & -4.0 \\
\hline Equus & stenonis & La Puebla de Valverde & PVAL & Teruel & MNCN & 2.1 & 1609 & M3 & 21 & Univ. Utrecht & -11.4 & -4.1 \\
\hline Equus & stenonis & La Puebla de Valverde & PVAL & Teruel & MNCN & 2.1 & 1609 & M3 & 26 & Univ. Utrecht & -11.3 & -3.7 \\
\hline Equus & stenonis & La Puebla de Valverde & PVAL & Teruel & MNCN & 2.1 & 1609 & M3 & 32 & Univ. Utrecht & -11.1 & -3.7 \\
\hline Equus & stenonis & La Puebla de Valverde & PVAL & Teruel & MNCN & 2.1 & 1609 & M3 & 40 & Univ. Utrecht & -11.0 & -4.3 \\
\hline Equus & stenonis & La Puebla de Valverde & PVAL & Teruel & MNCN & 2.1 & 1609 & M3 & 47 & Univ. Utrecht & -11.4 & -5.4 \\
\hline Equus & stenonis & La Puebla de Valverde & PVAL & Teruel & MNCN & 2.1 & 1609 & M3 & 53 & Univ. Utrecht & -11.6 & -4.5 \\
\hline Equus & stenonis & La Puebla de Valverde & PVAL & Teruel & MNCN & 2.1 & 1609 & M3 & 58 & Univ. Utrecht & -11.2 & -4.6 \\
\hline Hipparion & $s p$ & Orrios & OR & Teruel & MNCN & 4.4 & 212 & m3 & 5 & Univ. Utrecht & -11.6 & -3.3 \\
\hline Hipparion & sp. & Orrios & OR & Teruel & MNCN & 4.4 & 212 & $\mathrm{~m} 3$ & 8 & Univ. Utrecht & -11.4 & -3.2 \\
\hline Hipparion & sp. & Orrios & OR & Teruel & MNCN & 4.4 & 212 & m3 & 11 & Univ. Utrecht & -11.4 & -3.3 \\
\hline Hipparion & sp. & Orrios & OR & Teruel & MNCN & 4.4 & 212 & $\mathrm{~m} 3$ & 15 & Univ. Utrecht & -11.3 & -3.9 \\
\hline Hipparion & sp. & Orrios & OR & Teruel & MNCN & 4.4 & 212 & m3 & 18 & Univ. Utrecht & -11.3 & -4.0 \\
\hline Hipparion & sp. & Orrios & OR & Teruel & MNCN & 4.4 & 212 & m3 & 21 & Univ. Utrecht & -11.7 & -3.6 \\
\hline Hipparion & sp. & Orrios & OR & Teruel & MNCN & 4.4 & 212 & $\mathrm{~m} 3$ & 23 & Univ. Utrecht & -11.6 & -3.9 \\
\hline Hipparion & sp. & Orrios & OR & Teruel & MNCN & 4.4 & 212 & $\mathrm{~m} 3$ & 25 & Univ. Utrecht & -11.5 & -4.0 \\
\hline Hipparion & sp. & Orrios & OR & Teruel & MNCN & 4.4 & 212 & m3 & 27 & Univ. Utrecht & -11.6 & -3.8 \\
\hline Hipparion & sp. & Orrios & OR & Teruel & MNCN & 4.4 & 212 & m3 & 30 & Univ. Utrecht & -11.7 & -3.7 \\
\hline Hipparion & sp. & Orrios & OR & Teruel & MNCN & 4.4 & 212 & m3 & 32 & Univ. Utrecht & -11.6 & -3.5 \\
\hline Hipparion & sp. & Orrios & OR & Teruel & MNCN & 4.4 & 212 & m3 & 34 & Univ. Utrecht & -11.8 & -3.4 \\
\hline Hipparion & sp. & Orrios & OR & Teruel & MNCN & 4.4 & 212 & m3 & 36 & Univ. Utrecht & -11.6 & -3.7 \\
\hline Hipparion & sp. & Orrios & OR & Teruel & MNCN & 4.4 & 212 & $\mathrm{~m} 3$ & 39 & Univ. Utrecht & -11.7 & -4.3 \\
\hline Hipparion & sp. & Orrios & OR & Teruel & MNCN & 4.4 & 212 & m3 & 41 & Univ. Utrecht & -11.8 & -4.4 \\
\hline Hipparion & sp. & Orrios & OR & Teruel & MNCN & 4.4 & 212 & m3 & 43 & Univ. Utrecht & -11.6 & -3.9 \\
\hline Hipparion & sp. & Orrios & OR & Teruel & MNCN & 4.4 & 212 & m3 & 46 & Univ. Utrecht & -11.8 & -4.1 \\
\hline Hipparion & sp. & Orrios & OR & Teruel & MNCN & 4.4 & 212 & $\mathrm{~m} 3$ & 48 & Univ. Utrecht & -11.9 & -4.4 \\
\hline Hipparion & sp. & La Calera & LCA & Teruel & MNCN & $4.8-3.8$ & 81 & M3 & 4 & Univ. Utrecht & -11.3 & -3.2 \\
\hline Hipparion & sp. & La Calera & LCA & Teruel & MNCN & $4.8-3.8$ & 81 & M3 & 9 & Univ. Utrecht & -11.3 & -2.9 \\
\hline Hipparion & sp. & La Calera & LCA & Teruel & MNCN & $4.8-3.8$ & 81 & M3 & 13 & Univ. Utrecht & -11.4 & -2.9 \\
\hline Hipparion & sp. & La Calera & LCA & Teruel & MNCN & $4.8-3.8$ & 81 & M3 & 15 & Univ. Utrecht & -11.6 & -2.4 \\
\hline Hipparion & sp. & La Calera & LCA & Teruel & MNCN & $4.8-3.8$ & 81 & M3 & 18 & Univ. Utrecht & -11.7 & -2.4 \\
\hline Hipparion & sp. & La Calera & LCA & Teruel & MNCN & $4.8-3.8$ & 81 & M3 & 22 & Univ. Utrecht & -11.7 & -2.6 \\
\hline Hipparion & sp. & La Calera & LCA & Teruel & MNCN & $4.8-3.8$ & 81 & M3 & 24 & Univ. Utrecht & -11.6 & -2.8 \\
\hline Hipparion & sp. & La Calera & LCA & Teruel & MNCN & $4.8-3.8$ & 81 & M3 & 27 & Univ. Utrecht & -11.5 & -3.4 \\
\hline Hipparion & sp. & La Calera & LCA & Teruel & MNCN & $4.8-3.8$ & 81 & M3 & 30 & Univ. Utrecht & -11.4 & -4.1 \\
\hline Hipparion & sp. & La Calera & LCA & Teruel & MNCN & $4.8-3.8$ & 81 & M3 & 33 & Univ. Utrecht & -10.8 & -3.8 \\
\hline Hipparion & sp. & La Calera & LCA & Teruel & MNCN & $4.8-3.8$ & 1236 & M3 & Base & Univ. Utah & -11.2 & -2.9 \\
\hline Hipparion & sp. & La Calera & LCA & Teruel & MNCN & $4.8-3.8$ & 1277 & m3 & Base & Univ. Utah & -11.2 & -4.4 \\
\hline Hipparion & sp. & La Calera & LCA & Teruel & MNCN & $4.8-3.8$ & 1235 & $\mathrm{~m} 3$ & Base & Univ. Utah & -11.2 & -4.2 \\
\hline Hipparion & sp. & La Gloria 4 & GLO4 & Teruel & MNCN & $5.3-4.8$ & unn. (1) & $\mathrm{P} 2$ & 20 & Univ. Utrecht & -11.7 & -3.6 \\
\hline Hipparion & sp. & La Gloria 4 & GLO4 & Teruel & MNCN & $5.3-4.8$ & unn. (2) & $\mathrm{m} 2$ & Base & Univ. Utah & -12.0 & -4.0 \\
\hline Hipparion & sp. & La Gloria 4 & GLO4 & Teruel & MNCN & $5.3-4.8$ & 313 & $\mathrm{P} 2$ & Base & Univ. Utah & -11.9 & -3.8 \\
\hline Hipparion & sp. & Venta del Moro & VM & Cabriel & MNCN & 6.0 & 3164 & M3 & 4 & Univ. Utrecht & -10.7 & -3.3 \\
\hline Hipparion & sp. & Venta del Moro & VM & Cabriel & MNCN & 6.0 & 3164 & M3 & 7.5 & Univ. Utrecht & -11.1 & -3.1 \\
\hline Hipparion & sp. & Venta del Moro & VM & Cabriel & MNCN & 6.0 & 3164 & M3 & 10.5 & Univ. Utrecht & -10.9 & -2.7 \\
\hline Hipparion & sp. & Venta del Moro & VM & Cabriel & MNCN & 6.0 & 3164 & M3 & 12 & Univ. Utrecht & -10.9 & -2.4 \\
\hline Hipparion & sp. & Venta del Moro & VM & Cabriel & MNCN & 6.0 & 3164 & M3 & 15 & Univ. Utrecht & -10.6 & -2.1 \\
\hline Hipparion & sp. & Venta del Moro & VM & Cabriel & MNCN & 6.0 & 3164 & M3 & 17 & Univ. Utrecht & -10.7 & -1.9 \\
\hline Hipparion & sp. & Venta del Moro & VM & Cabriel & MNCN & 6.0 & 3164 & M3 & 20 & Univ. Utrecht & -10.1 & -1.7 \\
\hline Hipparion & sp. & Venta del Moro & VM & Cabriel & MNCN & 6.0 & 3164 & M3 & 21 & Univ. Utrecht & -10.2 & -1.1 \\
\hline Hipparion & sp. & Venta del Moro & VM & Cabriel & MNCN & 6.0 & 3164 & M3 & 26 & Univ. Utrecht & -10.0 & -1.3 \\
\hline Hipparion & sp. & Venta del Moro & VM & Cabriel & MNCN & 6.0 & 3164 & M3 & 27 & Univ. Utrecht & -10.2 & -1.5 \\
\hline Hipparion & sp. & Venta del Moro & VM & Cabriel & MNCN & 6.0 & 3164 & M3 & 28 & Univ. Utrecht & -10.1 & -1.5 \\
\hline Hipparion & sp. & Venta del Moro & VM & Cabriel & MNCN & 6.0 & 3164 & M3 & 29.5 & Univ. Utrecht & -10.2 & -1.7 \\
\hline Hipparion & sp. & Venta del Moro & VM & Cabriel & MNCN & 6.0 & 3164 & M3 & 31 & Univ. Utrecht & -10.0 & -1.7 \\
\hline Hipparion & sp. & Venta del Moro & VM & Cabriel & MNCN & 6.0 & 3164 & M3 & 33 & Univ. Utrecht & -9.9 & -1.3 \\
\hline Hipparion & sp. & Venta del Moro & VM & Cabriel & MNCN & 6.0 & 3164 & M3 & 37 & Univ. Utrecht & -9.8 & -1.6 \\
\hline Hipparion & sp. & Venta del Moro & VM & Cabriel & MNCN & 6.0 & 3164 & M3 & 40 & Univ. Utrecht & -9.9 & -1.8 \\
\hline Hipparion & sp. & Venta del Moro & VM & Cabriel & MNCN & 6.0 & 3164 & M3 & 44.5 & Univ. Utrecht & -10.0 & -2.2 \\
\hline Hipparion & sp. & Venta del Moro & VM & Cabriel & MNCN & 6.0 & 3164 & M3 & 48 & Univ. Utrecht & -10.3 & -2.0 \\
\hline Hipparion & periafricanum & Las Casiones & KS & Teruel & FCPD & $6.4-6.1$ & unn. & p3-m2 & 8 & Univ. Utrecht & -11.2 & -3.5 \\
\hline Hipparion & gromovae & Las Casiones & KS & Teruel & FCPD & $6.4-6.1$ & unn. & P3-M2 & 21 & Univ. Utrecht & -10.4 & -4.8 \\
\hline Hipparion & primigenium & Las Casiones & KS & Teruel & FCPD & $6.4-6.1$ & unn. & P3-M2 & 30 & Univ. Utrecht & -10.8 & -2.3 \\
\hline Hipparion & periafricanum & Las Casiones & KS & Teruel & FCPD & $6.4-6.1$ & 1048 & $\mathrm{~m} 3$ & Base & Univ. Utah & -11.6 & -5.4 \\
\hline Hipparion & gromovae & Las Casiones & KS & Teruel & FCPD & $6.4-6.1$ & 1432 & $\mathrm{~m} 3$ & Base & Univ. Utah & -10.8 & -4.4 \\
\hline Hipparion & primigenium & Las Casiones & KS & Teruel & FCPD & $6.4-6.1$ & 1277 & $\mathrm{~m} 3$ & Base & Univ. Utah & -10.8 & -4.8 \\
\hline Hipparion & gromovae & El Arquillo & ARQ & Teruel & ESUU & $6.4-6.1$ & unn. & M3 & 3 & Univ. Utrecht & -11.5 & -3.2 \\
\hline Hipparion & gromovae & El Arquillo & ARQ & Teruel & ESUU & $6.4-6.1$ & unn. & M3 & 6 & Univ. Utrecht & -11.3 & -3.2 \\
\hline Hipparion & gromovae & El Arquillo & ARQ & Teruel & ESUU & $6.4-6.1$ & unn. & M3 & 8 & Univ. Utrecht & -11.2 & -2.9 \\
\hline Hipparion & gromovae & El Arquillo & ARQ & Teruel & ESUU & $6.4-6.1$ & unn. & M3 & 10 & Univ. Utrecht & -11.5 & -3.1 \\
\hline Hipparion & gromovae & El Arquillo & ARQ & Teruel & ESUU & $6.4-6.1$ & unn. & M3 & 12 & Univ. Utrecht & -11.3 & -2.3 \\
\hline Hipparion & gromovae & El Arquillo & ARQ & Teruel & ESUU & $6.4-6.1$ & unn. & M3 & 15 & Univ. Utrecht & -11.4 & -2.6 \\
\hline Hipparion & gromovae & El Arquillo & ARQ & Teruel & ESUU & $6.4-6.1$ & unn. & M3 & 16.5 & Univ. Utrecht & -11.5 & -2.5 \\
\hline Hipparion & gromovae & El Arquillo & ARQ & Teruel & ESUU & $6.4-6.1$ & unn. & M3 & 19 & Univ. Utrecht & -10.9 & -1.8 \\
\hline
\end{tabular}


Table 1 (continued)

\begin{tabular}{|c|c|c|c|c|c|c|c|c|c|c|c|c|}
\hline Genus & Species & Locality & Locality code & Basin & Collection & Age (Ma) & Specimen & Element & $\mathrm{mm}$ from top & Laboratory & $\delta^{13} \mathrm{C}$ & $\delta^{18} \mathrm{O}$ \\
\hline Hipparion & gromovae & El Arquillo & ARQ & Teruel & ESUU & $6.4-6.1$ & unn. & M3 & 20.5 & Univ. Utrecht & -11.2 & -2.2 \\
\hline Hipparion & gromovae & El Arquillo & ARQ & Teruel & ESUU & $6.4-6.1$ & unn. & M3 & 22 & Univ. Utrecht & -11.0 & -2.4 \\
\hline Hipparion & gromovae & El Arquillo & ARQ & Teruel & ESUU & $6.4-6.1$ & unn. & M3 & 24 & Univ. Utrecht & -11.1 & -2.6 \\
\hline Hipparion & gromovae & El Arquillo & ARQ & Teruel & ESUU & $6.4-6.1$ & unn. & M3 & 26.5 & Univ. Utrecht & -10.9 & -2.9 \\
\hline Hipparion & gromovae & El Arquillo & ARQ & Teruel & ESUU & $6.4-6.1$ & unn. & M3 & 29 & Univ. Utrecht & -11.3 & -3.3 \\
\hline Hipparion & gromovae & El Arquillo & ARQ & Teruel & ESUU & $6.4-6.1$ & unn. & M3 & 33 & Univ. Utrecht & -11.1 & -3.9 \\
\hline Hipparion & gromovae & El Arquillo & ARQ & Teruel & ESUU & $6.4-6.1$ & unn. & M3 & 35 & Univ. Utrecht & -11.0 & -3.8 \\
\hline Hipparion & gromovae & El Arquillo & ARQ & Teruel & ESUU & $6.4-6.1$ & unn. & M3 & 36 & Univ. Utrecht & -11.2 & -4.4 \\
\hline Hipparion & gromovae & El Arquillo & ARQ & Teruel & ESUU & 6.4-6.1 & unn. & M3 & 37 & Univ. Utrecht & -11.3 & -4.1 \\
\hline Hipparion & gromovae & El Arquillo & ARQ & Teruel & ESUU & $6.4-6.1$ & unn. & M3 & 38.5 & Univ. Utrecht & -11.3 & -2.5 \\
\hline Hipparion & primigenium & El Arquillo & ARQ & Teruel & MNCN & $6.4-6.1$ & 31986 & M2 & Base & Univ. Utah & -11.3 & -3.0 \\
\hline Hipparion & primigenium & El Arquillo & ARQ & Teruel & MNCN & $6.4-6.1$ & 31986 & M2 & Base & Univ. Utah & -10.9 & -3.7 \\
\hline Hipparion & primigenium & El Arquillo & ARQ & Teruel & MNCN & $6.4-6.1$ & 8879 & m3 & Base & Univ. Utah & -11.1 & -2.3 \\
\hline Hipparion & primigenium & Milagros & MIL & Teruel & FCPD & $6.4-6.1$ & unn. & M3 & Base & Univ. Utah & -11.2 & -3.5 \\
\hline Hipparion & primigenium & Milagros & MIL & Teruel & FCPD & $6.4-6.1$ & unn. & $\mathrm{m} 3$ & Base & Univ. Utah & -11.2 & -2.6 \\
\hline Hipparion & concudense & Los Mansuetos & LM & Teruel & ESUU & $7.2-7.1$ & 1108 & m3 & 23 & Univ. Utrecht & -11.0 & -4.4 \\
\hline Hipparion & concudense & Los Mansuetos & LM & Teruel & ESUU & $7.2-7.1$ & 1108 & $\mathrm{~m} 3$ & 33 & Univ. Utrecht & -11.0 & -6.6 \\
\hline Hipparion & concudense & Los Mansuetos & LM & Teruel & MNCN & $7.2-7.1$ & unn. & M2 & Base & Univ. Utah & -10.7 & -1.7 \\
\hline Hipparion & concudense & Los Mansuetos & LM & Teruel & MNCN & $7.2-7.1$ & unn. & M2 & Base & Univ. Utah & -10.7 & -3.7 \\
\hline Hipparion & concudense & Concud C. de la Garita & $\mathrm{CC}$ & Teruel & ESUU & $7.2-7.1$ & 117 & $\mathrm{~m} 3$ & 31 & Univ. Utrecht & -10.1 & -5.5 \\
\hline Hipparion & concudense & Concud C. de la Garita & $\mathrm{CC}$ & Teruel & ESUU & $7.2-7.1$ & 117 & $\mathrm{~m} 3$ & 40 & Univ. Utrecht & -10.2 & -5.1 \\
\hline Hipparion & concudense & Concud C. de la Garita & $\mathrm{CC}$ & Teruel & ESUU & $7.2-7.1$ & 277 & M3 & 1 & Univ. Utrecht & -10.3 & -2.5 \\
\hline Hipparion & concudense & Concud C. de la Garita & $\mathrm{CC}$ & Teruel & ESUU & $7.2-7.1$ & 277 & M3 & 5.5 & Univ. Utrecht & -10.1 & -3.5 \\
\hline Hipparion & concudense & Concud C. de la Garita & $\mathrm{CC}$ & Teruel & ESUU & $7.2-7.1$ & 277 & M3 & 10 & Univ. Utrecht & -10.3 & -3.1 \\
\hline Hipparion & concudense & Concud C. de la Garita & $\mathrm{CC}$ & Teruel & ESUU & $7.2-7.1$ & 277 & M3 & 12 & Univ. Utrecht & -9.9 & -3.9 \\
\hline Hipparion & concudense & Concud C. de la Garita & $\mathrm{CC}$ & Teruel & ESUU & $7.2-7.1$ & 277 & M3 & 14 & Univ. Utrecht & -10.0 & -4.1 \\
\hline Hipparion & concudense & Concud C. de la Garita & $\mathrm{CC}$ & Teruel & ESUU & $7.2-7.1$ & 277 & M3 & 16 & Univ. Utrecht & -9.7 & -4.2 \\
\hline Hipparion & concudense & Concud C. de la Garita & $\mathrm{CC}$ & Teruel & ESUU & $7.2-7.1$ & 277 & M3 & 18 & Univ. Utrecht & -9.8 & -3.4 \\
\hline Hipparion & concudense & Concud C. de la Garita & $\mathrm{CC}$ & Teruel & ESUU & $7.2-7.1$ & 277 & M3 & 19.5 & Univ. Utrecht & -9.7 & -3.3 \\
\hline Hipparion & concudense & Concud C. de la Garita & $\mathrm{CC}$ & Teruel & ESUU & $7.2-7.1$ & 277 & M3 & 21 & Univ. Utrecht & -10.0 & -3.3 \\
\hline Hipparion & concudense & Concud C. de la Garita & $\mathrm{CC}$ & Teruel & ESUU & $7.2-7.1$ & 277 & M3 & 23 & Univ. Utrecht & -10.1 & -3.9 \\
\hline Hipparion & concudense & Concud C. de la Garita & $\mathrm{CC}$ & Teruel & ESUU & $7.2-7.1$ & 277 & M3 & 25 & Univ. Utrecht & -10.2 & -3.4 \\
\hline Hipparion & concudense & Concud C. de la Garita & $\mathrm{CC}$ & Teruel & ESUU & $7.2-7.1$ & 277 & M3 & 27 & Univ. Utrecht & -10.2 & -3.6 \\
\hline Hipparion & concudense & Concud C. de la Garita & $\mathrm{CC}$ & Teruel & ESUU & $7.2-7.1$ & 277 & M3 & 30.5 & Univ. Utrecht & -10.2 & -3.2 \\
\hline Hipparion & concudense & Concud C. de la Garita & $\mathrm{CC}$ & Teruel & ESUU & $7.2-7.1$ & 277 & M3 & 32 & Univ. Utrecht & -10.7 & -2.5 \\
\hline Hipparion & concudense & Concud C. de la Garita & $\mathrm{CC}$ & Teruel & ESUU & $7.2-7.1$ & 277 & M3 & 34 & Univ. Utrecht & -10.7 & -2.1 \\
\hline Hipparion & concudense & Concud C. de la Garita & $\mathrm{CC}$ & Teruel & ESUU & $7.2-7.1$ & 277 & M3 & 36 & Univ. Utrecht & -10.5 & -2.2 \\
\hline Hipparion & concudense & Concud C. de la Garita & $\mathrm{CC}$ & Teruel & ESUU & $7.2-7.1$ & 277 & M3 & 38 & Univ. Utrecht & -10.4 & -2.3 \\
\hline Hipparion & concudense & Concud C. de la Garita & $\mathrm{CC}$ & Teruel & ESUU & $7.2-7.1$ & 277 & M3 & 40 & Univ. Utrecht & -10.3 & -2.4 \\
\hline Hipparion & concudense & Concud C. de la Garita & $\mathrm{CC}$ & Teruel & MNCN & $7.2-7.1$ & 10585 & $\mathrm{~m} 3$ & Base & Univ. Utah & -10.9 & -3.7 \\
\hline Hipparion & concudense & Concud C. de la Garita & $\mathrm{CC}$ & Teruel & MNCN & $7.2-7.1$ & 10584 & $\mathrm{~m} 3$ & Base & Univ. Utah & -10.1 & -0.4 \\
\hline Hipparion & primigenium & Puente Minero & PM & Teruel & FCPD & $8.7-8.3$ & 596 & M3 & 17 & Univ. Utrecht & -11.3 & -3.9 \\
\hline Hipparion & primigenium & Puente Minero & PM & Teruel & FCPD & $8.7-8.3$ & 596 & M3 & 27 & Univ. Utrecht & -11.1 & -4.3 \\
\hline Hipparion & primigenium & Puente Minero & PM & Teruel & FCPD & $8.7-8.3$ & 212 & $\mathrm{~m} 3$ & 1.5 & Univ. Utrecht & -10.5 & -4.0 \\
\hline Hipparion & primigenium & Puente Minero & PM & Teruel & FCPD & $8.7-8.3$ & 212 & $\mathrm{~m} 3$ & 4.5 & Univ. Utrecht & -10.6 & -4.2 \\
\hline Hipparion & primigenium & Puente Minero & PM & Teruel & FCPD & $8.7-8.3$ & 212 & $\mathrm{~m} 3$ & 5.5 & Univ. Utrecht & -10.6 & -4.0 \\
\hline Hipparion & primigenium & Puente Minero & PM & Teruel & FCPD & $8.7-8.3$ & 212 & $\mathrm{~m} 3$ & 6.5 & Univ. Utrecht & -10.6 & -3.7 \\
\hline Hipparion & primigenium & Puente Minero & PM & Teruel & FCPD & $8.7-8.3$ & 212 & $\mathrm{~m} 3$ & 8 & Univ. Utrecht & -10.7 & -3.7 \\
\hline Hipparion & primigenium & Puente Minero & PM & Teruel & FCPD & $8.7-8.3$ & 212 & $\mathrm{~m} 3$ & 9.5 & Univ. Utrecht & -10.6 & -3.2 \\
\hline Hipparion & primigenium & Puente Minero & PM & Teruel & FCPD & $8.7-8.3$ & 212 & $\mathrm{~m} 3$ & 14 & Univ. Utrecht & -10.5 & -2.8 \\
\hline Hipparion & primigenium & Puente Minero & PM & Teruel & FCPD & $8.7-8.3$ & 212 & $\mathrm{~m} 3$ & 15.5 & Univ. Utrecht & -10.4 & -2.3 \\
\hline Hipparion & primigenium & Puente Minero & PM & Teruel & FCPD & $8.7-8.3$ & 212 & m3 & 17 & Univ. Utrecht & -10.4 & -3.3 \\
\hline Hipparion & primigenium & Puente Minero & PM & Teruel & FCPD & $8.7-8.3$ & 212 & $\mathrm{~m} 3$ & 19.5 & Univ. Utrecht & -10.2 & -2.7 \\
\hline Hipparion & primigenium & Puente Minero & PM & Teruel & FCPD & $8.7-8.3$ & 212 & $\mathrm{~m} 3$ & 21.5 & Univ. Utrecht & -10.2 & -2.4 \\
\hline Hipparion & primigenium & Puente Minero & PM & Teruel & FCPD & $8.7-8.3$ & 212 & m3 & 24 & Univ. Utrecht & -10.3 & -3.2 \\
\hline Hipparion & primigenium & Puente Minero & PM & Teruel & FCPD & $8.7-8.3$ & 212 & $\mathrm{~m} 3$ & 26 & Univ. Utrecht & -10.4 & -2.9 \\
\hline Hipparion & primigenium & Puente Minero & PM & Teruel & FCPD & $8.7-8.3$ & 212 & $\mathrm{~m} 3$ & 27.5 & Univ. Utrecht & -10.6 & -3.1 \\
\hline Hipparion & primigenium & Puente Minero & PM & Teruel & FCPD & $8.7-8.3$ & 212 & $\mathrm{~m} 3$ & 29.5 & Univ. Utrecht & -10.6 & -3.0 \\
\hline Hipparion & primigenium & Puente Minero & PM & Teruel & FCPD & $8.7-8.3$ & 212 & $\mathrm{~m} 3$ & 31 & Univ. Utrecht & -10.7 & -3.8 \\
\hline Hipparion & primigenium & Puente Minero & PM & Teruel & FCPD & $8.7-8.3$ & 212 & $\mathrm{~m} 3$ & 32 & Univ. Utrecht & -10.7 & -4.1 \\
\hline Hipparion & primigenium & Puente Minero & PM & Teruel & FCPD & $8.7-8.3$ & 212 & $\mathrm{~m} 3$ & 33 & Univ. Utrecht & -10.8 & -3.8 \\
\hline Hipparion & primigenium & Puente Minero & PM & Teruel & FCPD & $8.7-8.3$ & 212 & m3 & 34.5 & Univ. Utrecht & -10.9 & -4.2 \\
\hline Hipparion & primigenium & Puente Minero & PM & Teruel & FCPD & $8.7-8.3$ & 212 & $\mathrm{~m} 3$ & 36 & Univ. Utrecht & -10.8 & -3.4 \\
\hline Hipparion & primigenium & Puente Minero & PM & Teruel & FCPD & $8.7-8.3$ & 212 & $\mathrm{~m} 3$ & 37.5 & Univ. Utrecht & -10.7 & -2.8 \\
\hline Hipparion & primigenium & Puente Minero & PM & Teruel & FCPD & $8.7-8.3$ & 212 & $\mathrm{~m} 3$ & 39 & Univ. Utrecht & -10.7 & -3.4 \\
\hline Hipparion & primigenium & Puente Minero & PM & Teruel & FCPD & $8.7-8.3$ & 212 & m3 & 40.5 & Univ. Utrecht & -10.9 & -2.1 \\
\hline Hipparion & primigenium & Puente Minero & PM & Teruel & FCPD & $8.7-8.3$ & 212 & $\mathrm{~m} 3$ & 41.5 & Univ. Utrecht & -11.1 & -2.5 \\
\hline Hipparion & primigenium & Puente Minero & PM & Teruel & FCPD & $8.7-8.3$ & 212 & $\mathrm{~m} 3$ & 42.5 & Univ. Utrecht & -11.0 & -2.2 \\
\hline Hipparion & primigenium & Puente Minero & PM & Teruel & FCPD & $8.7-8.3$ & 283 & $\mathrm{~m} 3$ & Base & Univ. Utah & -10.7 & -1.6 \\
\hline Hipparion & primigenium & Puente Minero & PM & Teruel & FCPD & $8.7-8.3$ & 209 & $\mathrm{~m} 3$ & Base & Univ. Utah & -10.9 & -2.0 \\
\hline Hipparion & primigenium & Puente Minero & PM & Teruel & FCPD & $8.7-8.3$ & 730 & $\mathrm{~m} 3$ & Base & Univ. Utah & -10.8 & -3.4 \\
\hline Hipparion & laromae & La Roma 2 & $\mathrm{R} 2$ & Teruel & FCPD & $9.3-9.2$ & 18 & $\mathrm{~m} 3$ & 28 & Univ. Utrecht & -10.7 & -2.7 \\
\hline Hipparion & laromae & La Roma 2 & $\mathrm{R} 2$ & Teruel & FCPD & $9.3-9.2$ & 18 & m3 & 37 & Univ. Utrecht & -10.3 & -3.5 \\
\hline Hipparion & laromae & La Roma 2 & $\mathrm{R} 2$ & Teruel & FCPD & $9.3-9.2$ & 2065 & M3 & 1.5 & Univ. Utrecht & -10.5 & -3.4 \\
\hline Hipparion & laromae & La Roma 2 & $\mathrm{R} 2$ & Teruel & FCPD & $9.3-9.2$ & 2065 & M3 & 3.5 & Univ. Utrecht & -10.5 & -3.1 \\
\hline
\end{tabular}


Table 1 (continued)

\begin{tabular}{|c|c|c|c|c|c|c|c|c|c|c|c|c|}
\hline Genus & Species & Locality & Locality code & Basin & Collection & Age (Ma) & Specimen & Element & $\mathrm{mm}$ from top & Laboratory & $\delta^{13} \mathrm{C}$ & $\delta^{18} \mathrm{O}$ \\
\hline Hipparion & laromae & La Roma 2 & R2 & Teruel & FCPD & $9.3-9.2$ & 2065 & M3 & 5.5 & Univ. Utrecht & -10.6 & -3.4 \\
\hline Hipparion & laromae & La Roma 2 & R2 & Teruel & FCPD & $9.3-9.2$ & 2065 & M3 & 8 & Univ. Utrecht & -10.6 & -2.9 \\
\hline Hipparion & laromae & La Roma 2 & R2 & Teruel & FCPD & $9.3-9.2$ & 2065 & M3 & 10.5 & Univ. Utrecht & -10.4 & -2.8 \\
\hline Hipparion & laromae & La Roma 2 & R2 & Teruel & FCPD & $9.3-9.2$ & 2065 & M3 & 13.5 & Univ. Utrecht & -10.5 & -2.8 \\
\hline Hipparion & laromae & La Roma 2 & R2 & Teruel & FCPD & $9.3-9.2$ & 2065 & M3 & 17 & Univ. Utrecht & -10.1 & -2.3 \\
\hline Hipparion & laromae & La Roma 2 & R2 & Teruel & FCPD & $9.3-9.2$ & 2065 & M3 & 20 & Univ. Utrecht & -10.1 & -1.6 \\
\hline Hipparion & laromae & La Roma 2 & R2 & Teruel & FCPD & $9.3-9.2$ & 2065 & M3 & 25 & Univ. Utrecht & -9.9 & -2.0 \\
\hline Hipparion & laromae & La Roma 2 & R2 & Teruel & FCPD & $9.3-9.2$ & 2065 & M3 & 27 & Univ. Utrecht & -9.7 & -1.1 \\
\hline Hipparion & laromae & La Roma 2 & R2 & Teruel & FCPD & $9.3-9.2$ & 2065 & M3 & 30 & Univ. Utrecht & -9.6 & -1.0 \\
\hline Hipparion & laromae & La Roma 2 & R2 & Teruel & FCPD & $9.3-9.2$ & 2065 & M3 & 33 & Univ. Utrecht & -9.5 & -0.5 \\
\hline Hipparion & laromae & La Roma 2 & R2 & Teruel & FCPD & $9.3-9.2$ & 2065 & M3 & 35 & Univ. Utrecht & -9.4 & -0.8 \\
\hline Hipparion & laromae & La Roma 2 & R2 & Teruel & FCPD & $9.3-9.2$ & 2065 & M3 & 38 & Univ. Utrecht & -9.3 & -0.6 \\
\hline Hipparion & laromae & La Roma 2 & R2 & Teruel & FCPD & $9.3-9.2$ & 2065 & M3 & 41 & Univ. Utrecht & -9.4 & -1.4 \\
\hline Hipparion & laromae & La Roma 2 & R2 & Teruel & FCPD & $9.3-9.2$ & 2065 & M3 & 45 & Univ. Utrecht & -9.5 & -1.8 \\
\hline Hipparion & laromae & La Roma 2 & R2 & Teruel & FCPD & $9.3-9.2$ & 2065 & M3 & 47.5 & Univ. Utrecht & -9.5 & -2.1 \\
\hline Hipparion & laromae & La Roma 2 & R2 & Teruel & FCPD & $9.3-9.2$ & 2065 & M3 & 49.5 & Univ. Utrecht & -9.5 & -2.4 \\
\hline Hipparion & laromae & La Roma 2 & R2 & Teruel & FCPD & $9.3-9.2$ & 2065 & M3 & 53 & Univ. Utrecht & -9.3 & -2.1 \\
\hline Hipparion & laromae & La Roma 2 & R2 & Teruel & FCPD & $9.3-9.2$ & 2065 & M3 & Base & Univ. Utah & -9.6 & -1.0 \\
\hline Hipparion & primigenium & Masía del Barbo 2B & MBB & Teruel & ESUU & 9.6 & 78 & $\mathrm{~m} 3$ & 11 & Univ. Utrecht & -10.0 & -4.3 \\
\hline Hipparion & primigenium & Masía del Barbo 2B & MBB & Teruel & ESUU & 9.6 & 78 & $\mathrm{~m} 3$ & 19 & Univ. Utrecht & -10.2 & -3.3 \\
\hline Hipparion & primigenium & Masía del Barbo 2A & MBA & Teruel & ESUU & 9.7 & 52 & m3 & 5 & Univ. Utrecht & -11.0 & 0.8 \\
\hline Hipparion & primigenium & Masía del Barbo 2A & MBA & Teruel & ESUU & 9.7 & 52 & $\mathrm{~m} 3$ & 9 & Univ. Utrecht & -11.0 & 0.5 \\
\hline Hipparion & primigenium & Masía del Barbo 2A & MBA & Teruel & ESUU & 9.7 & 52 & $\mathrm{~m} 3$ & 14 & Univ. Utrecht & -11.0 & 0.7 \\
\hline Hipparion & primigenium & Masía del Barbo 2A & MBA & Teruel & ESUU & 9.7 & 52 & $\mathrm{~m} 3$ & 17 & Univ. Utrecht & -10.8 & 0.4 \\
\hline Hipparion & primigenium & Masía del Barbo 2A & MBA & Teruel & ESUU & 9.7 & 52 & $\mathrm{~m} 3$ & 17.5 & Univ. Utrecht & -10.6 & 1.0 \\
\hline Hipparion & primigenium & Masía del Barbo 2A & MBA & Teruel & ESUU & 9.7 & 52 & $\mathrm{~m} 3$ & 22.5 & Univ. Utrecht & -10.7 & 0.3 \\
\hline Hipparion & primigenium & Masía del Barbo 2A & MBA & Teruel & ESUU & 9.7 & 52 & $\mathrm{~m} 3$ & 27 & Univ. Utrecht & -10.5 & -0.9 \\
\hline Hipparion & primigenium & Masía del Barbo 2A & MBA & Teruel & ESUU & 9.7 & 52 & $\mathrm{~m} 3$ & 29 & Univ. Utrecht & -10.3 & -0.8 \\
\hline Hipparion & primigenium & Masía del Barbo 2A & MBA & Teruel & ESUU & 9.7 & 52 & $\mathrm{~m} 3$ & 30.5 & Univ. Utrecht & -10.3 & -1.0 \\
\hline Hipparion & primigenium & Masía del Barbo 2A & MBA & Teruel & ESUU & 9.7 & 52 & $\mathrm{~m} 3$ & 32.5 & Univ. Utrecht & -10.2 & -1.1 \\
\hline Hipparion & primigenium & Masía del Barbo 2A & MBA & Teruel & ESUU & 9.7 & 52 & $\mathrm{~m} 3$ & 34.5 & Univ. Utrecht & -10.2 & -1.0 \\
\hline Hipparion & primigenium & Masía del Barbo 2A & MBA & Teruel & ESUU & 9.7 & 52 & $\mathrm{~m} 3$ & 37 & Univ. Utrecht & -10.0 & -0.5 \\
\hline Hipparion & primigenium & Masía del Barbo 2A & MBA & Teruel & ESUU & 9.7 & 52 & $\mathrm{~m} 3$ & 39 & Univ. Utrecht & -9.8 & 0.6 \\
\hline Hipparion & primigenium & Masía del Barbo 2A & MBA & Teruel & ESUU & 9.7 & 52 & $\mathrm{~m} 3$ & 40.5 & Univ. Utrecht & -9.7 & 0.3 \\
\hline Hipparion & primigenium & Masía del Barbo 2A & MBA & Teruel & ESUU & 9.7 & 52 & $\mathrm{~m} 3$ & 43 & Univ. Utrecht & -9.7 & -0.7 \\
\hline Hipparion & primigenium & Masía del Barbo 2A & MBA & Teruel & ESUU & 9.7 & 52 & $\mathrm{~m} 3$ & 44.5 & Univ. Utrecht & -9.7 & -0.8 \\
\hline Hipparion & primigenium & Alto de Marinezquita & ALM & Teruel & ESUU & $9.8-9.9$ & unn. & $\mathrm{m} 3$ & 16 & Univ. Utrecht & -11.1 & -1.3 \\
\hline Hipparion & primigenium & Alto de Marinezquita & ALM & Teruel & ESUU & $9.8-9.9$ & unn. & $\mathrm{m} 3$ & 23 & Univ. Utrecht & -10.5 & -0.2 \\
\hline Hipparion & primigenium & Pedregueras $2 \mathrm{~A}$ & PED2A & C.-Daroca & ESUU & 10.0 & unn. & M1-2 & 2 & Univ. Utrecht & -10.7 & -2.6 \\
\hline Hipparion & primigenium & Pedregueras 2A & PED2A & C.-Daroca & ESUU & 10.0 & unn. & M1-2 & 4 & Univ. Utrecht & -10.5 & -2.2 \\
\hline Hipparion & primigenium & Pedregueras 2A & PED2A & C.-Daroca & ESUU & 10.0 & unn. & M1-2 & 8 & Univ. Utrecht & -10.4 & -2.5 \\
\hline Hipparion & primigenium & Pedregueras 2A & PED2A & C.-Daroca & ESUU & 10.0 & unn. & M1-2 & 12.5 & Univ. Utrecht & -10.4 & 0.1 \\
\hline Hipparion & primigenium & Pedregueras $2 \mathrm{~A}$ & PED2A & C.-Daroca & ESUU & 10.0 & unn. & M1-2 & 14.5 & Univ. Utrecht & -10.4 & -0.2 \\
\hline Hipparion & primigenium & Pedregueras 2A & PED2A & C.-Daroca & ESUU & 10.0 & unn. & M1-2 & 16.5 & Univ. Utrecht & -10.2 & 0.0 \\
\hline Hipparion & primigenium & Pedregueras $2 \mathrm{~A}$ & PED2A & C.-Daroca & ESUU & 10.0 & unn. & M1-2 & 18 & Univ. Utrecht & -10.1 & 0.3 \\
\hline Hipparion & primigenium & Pedregueras $2 \mathrm{~A}$ & PED2A & C.-Daroca & ESUU & 10.0 & unn. & M1-2 & 20 & Univ. Utrecht & -10.2 & 0.2 \\
\hline Hipparion & primigenium & Pedregueras 2A & PED2A & C.-Daroca & ESUU & 10.0 & unn. & M1-2 & 22 & Univ. Utrecht & -10.3 & -1.9 \\
\hline Hipparion & primigenium & Pedregueras 2A & PED2A & C.-Daroca & ESUU & 10.0 & unn. & M1-2 & 23.5 & Univ. Utrecht & -10.4 & -0.6 \\
\hline Hipparion & primigenium & Pedregueras $2 \mathrm{~A}$ & PED2A & C.-Daroca & ESUU & 10.0 & unn. & M1-2 & 25.5 & Univ. Utrecht & -10.3 & -1.1 \\
\hline Hipparion & primigenium & Pedregueras 2A & PED2A & C.-Daroca & ESUU & 10.0 & unn. & $\mathrm{p} 3 / 4$ & 27 & Univ. Utrecht & -11.2 & -3.6 \\
\hline Hipparion & primigenium & Pedregueras 2A & PED2A & C.-Daroca & ESUU & 10.0 & unn. & M1-2 & 27.5 & Univ. Utrecht & -10.4 & -1.1 \\
\hline Hipparion & primigenium & Pedregueras $2 \mathrm{~A}$ & PED2A & C.-Daroca & ESUU & 10.0 & unn. & M1-2 & 29.5 & Univ. Utrecht & -10.4 & -0.8 \\
\hline Hipparion & primigenium & Pedregueras $2 \mathrm{~A}$ & PED2A & C.-Daroca & ESUU & 10.0 & unn. & M1-2 & 31 & Univ. Utrecht & -10.5 & -1.0 \\
\hline Hipparion & primigenium & Pedregueras 2A & PED2A & C.-Daroca & ESUU & 10.0 & unn. & M1-2 & 33 & Univ. Utrecht & -10.5 & -1.6 \\
\hline Hipparion & primigenium & Pedregueras $2 \mathrm{~A}$ & PED2A & C.-Daroca & ESUU & 10.0 & unn. & M1-2 & 35 & Univ. Utrecht & -10.5 & -1.9 \\
\hline Hipparion & primigenium & Pedregueras $2 \mathrm{~A}$ & PED2A & C.-Daroca & ESUU & 10.0 & unn. & M1-2 & 36.5 & Univ. Utrecht & -10.4 & -1.7 \\
\hline Hipparion & primigenium & Pedregueras 2A & PED2A & C.-Daroca & ESUU & 10.0 & unn. & M1-2 & 38.5 & Univ. Utrecht & -10.4 & -2.0 \\
\hline Hipparion & primigenium & Pedregueras $2 \mathrm{~A}$ & PED2A & C.-Daroca & ESUU & 10.0 & unn. & p3/4 & 39 & Univ. Utrecht & -11.4 & -3.2 \\
\hline Hipparion & primigenium & Pedregueras $2 \mathrm{~A}$ & PED2A & C.-Daroca & ESUU & 10.0 & unn. & M1-2 & 40 & Univ. Utrecht & -10.3 & -2.0 \\
\hline Hipparion & primigenium & Pedregueras 2A & PED2A & C.-Daroca & ESUU & 10.0 & unn. & M1-2 & 42 & Univ. Utrecht & -10.3 & -2.0 \\
\hline Hipparion & primigenium & Pedregueras $2 \mathrm{~A}$ & PED2A & C.-Daroca & ESUU & 10.0 & unn. & M1-2 & 44 & Univ. Utrecht & -10.1 & -1.3 \\
\hline Hipparion & primigenium & Nombrevilla & NOM & C.-Daroca & ESUU & 10.8 & 1188 & M3 & 2 & Univ. Utrecht & -10.2 & -2.7 \\
\hline Hipparion & primigenium & Nombrevilla & NOM & C.-Daroca & ESUU & 10.8 & 1188 & M3 & 4 & Univ. Utrecht & -10.4 & -2.6 \\
\hline Hipparion & primigenium & Nombrevilla & NOM & C.-Daroca & ESUU & 10.8 & 1188 & M3 & 7 & Univ. Utrecht & -10.4 & -2.8 \\
\hline Hipparion & primigenium & Nombrevilla & NOM & C.-Daroca & ESUU & 10.8 & 1188 & M3 & 9.5 & Univ. Utrecht & -10.6 & -3.4 \\
\hline Hipparion & primigenium & Nombrevilla & NOM & C.-Daroca & ESUU & 10.8 & 1188 & M3 & 14 & Univ. Utrecht & -10.3 & -3.4 \\
\hline Hipparion & primigenium & Nombrevilla & NOM & C.-Daroca & ESUU & 10.8 & 1188 & M3 & 19 & Univ. Utrecht & -10.2 & -2.1 \\
\hline Hipparion & primigenium & Nombrevilla & NOM & C.-Daroca & ESUU & 10.8 & 1188 & M3 & 21 & Univ. Utrecht & -10.7 & -1.9 \\
\hline Hipparion & primigenium & Nombrevilla & NOM & C.-Daroca & ESUU & 10.8 & 1188 & M3 & 22 & Univ. Utrecht & -10.3 & -2.5 \\
\hline Hipparion & primigenium & Nombrevilla & NOM & C.-Daroca & ESUU & 10.8 & 1188 & M3 & 23.5 & Univ. Utrecht & -10.3 & -2.5 \\
\hline Hipparion & primigenium & Nombrevilla & NOM & C.-Daroca & ESUU & 10.8 & 1188 & M3 & 25.5 & Univ. Utrecht & -10.6 & -1.9 \\
\hline Hipparion & primigenium & Nombrevilla & NOM & C.-Daroca & ESUU & 10.8 & 1188 & M3 & 27 & Univ. Utrecht & -10.6 & -1.8 \\
\hline Hipparion & primigenium & Nombrevilla & NOM & C.-Daroca & ESUU & 10.8 & 1188 & M3 & 30 & Univ. Utrecht & -10.6 & -2.0 \\
\hline Hipparion & primigenium & Nombrevilla & NOM & C.-Daroca & ESUU & 10.8 & 1188 & M3 & 32 & Univ. Utrecht & -10.5 & -2.2 \\
\hline Hipparion & primigenium & Nombrevilla & NOM & C.-Daroca & ESUU & 10.8 & 1188 & M3 & 33.5 & Univ. Utrecht & -10.1 & -1.4 \\
\hline Hipparion & primigenium & Nombrevilla & NOM & C.-Daroca & ESUU & 10.8 & 1188 & M3 & 34.5 & Univ. Utrecht & -10.2 & -2.8 \\
\hline Hipparion & primigenium & Nombrevilla & NOM & C.-Daroca & ESUU & 10.8 & 1188 & M3 & 36.5 & Univ. Utrecht & -10.3 & -2.3 \\
\hline
\end{tabular}


Table 1 (continued)

\begin{tabular}{|c|c|c|c|c|c|c|c|c|c|c|c|c|}
\hline Genus & Species & Locality & Locality code & Basin & Collection & Age (Ma) & Specimen & Element & $\mathrm{mm}$ from top & Laboratory & $\delta^{13} \mathrm{C}$ & $\delta^{18} \mathrm{O}$ \\
\hline Hipparion & primigenium & Nombrevilla & NOM & C.-Daroca & ESUU & 10.8 & 1188 & M3 & 38.5 & Univ. Utrecht & -10.2 & -2.1 \\
\hline Hipparion & primigenium & Nombrevilla & NOM & C.-Daroca & ESUU & 10.8 & 1188 & M3 & 40 & Univ. Utrecht & -10.3 & -2.0 \\
\hline Hipparion & primigenium & Nombrevilla & NOM & C.-Daroca & ESUU & 10.8 & 1188 & M3 & 42.5 & Univ. Utrecht & -10.0 & -1.6 \\
\hline Hipparion & primigenium & Nombrevilla & NOM & C.-Daroca & ESUU & 10.8 & 1188 & M3 & 44.5 & Univ. Utrecht & -10.1 & -2.7 \\
\hline Hipparion & primigenium & Nombrevilla & NOM & C.-Daroca & ESUU & 10.8 & 1188 & M3 & 47 & Univ. Utrecht & -9.6 & -3.0 \\
\hline Hipparion & primigenium & Nombrevilla & NOM & C.-Daroca & ESUU & 10.8 & 1188 & M3 & 48.5 & Univ. Utrecht & -10.4 & -2.6 \\
\hline Hipparion & primigenium & Nombrevilla & NOM & C.-Daroca & ESUU & 10.8 & 1188 & M3 & 51.5 & Univ. Utrecht & -9.9 & -2.5 \\
\hline Hipparion & primigenium & Nombrevilla & NOM & C.-Daroca & ESUU & 10.8 & 1188 & M3 & 53.5 & Univ. Utrecht & -9.9 & -2.9 \\
\hline Hipparion & primigenium & Nombrevilla & NOM & C.-Daroca & ESUU & 10.8 & 1290 & $\mathrm{~m} 3$ & 5 & Univ. Utrecht & -11.4 & -2.5 \\
\hline Hipparion & primigenium & Nombrevilla & NOM & C.-Daroca & ESUU & 10.8 & 1290 & $\mathrm{~m} 3$ & 11 & Univ. Utrecht & -10.4 & -2.4 \\
\hline Hipparion & primigenium & Nombrevilla & NOM & C.-Daroca & ESUU & 10.8 & 1290 & $\mathrm{~m} 3$ & 14 & Univ. Utrecht & -10.5 & -2.5 \\
\hline Hipparion & primigenium & Nombrevilla & NOM & C.-Daroca & ESUU & 10.8 & 1290 & m3 & 15 & Univ. Utrecht & -10.6 & -3.0 \\
\hline Hipparion & primigenium & Nombrevilla & NOM & C.-Daroca & ESUU & 10.8 & 1290 & m3 & 17.5 & Univ. Utrecht & -10.4 & -3.1 \\
\hline Hipparion & primigenium & Nombrevilla & NOM & C.-Daroca & ESUU & 10.8 & 1290 & $\mathrm{~m} 3$ & 25 & Univ. Utrecht & -10.3 & -2.7 \\
\hline Hipparion & primigenium & Nombrevilla & NOM & C.-Daroca & ESUU & 10.8 & 1290 & $\mathrm{~m} 3$ & 26.5 & Univ. Utrecht & -10.4 & -2.7 \\
\hline Hipparion & primigenium & Nombrevilla & NOM & C.-Daroca & ESUU & 10.8 & 1290 & m3 & 29 & Univ. Utrecht & -10.4 & -2.6 \\
\hline Hipparion & primigenium & Nombrevilla & NOM & C.-Daroca & ESUU & 10.8 & 1290 & $\mathrm{~m} 3$ & 33 & Univ. Utrecht & -10.4 & -2.5 \\
\hline Hipparion & primigenium & Nombrevilla & NOM & C.-Daroca & ESUU & 10.8 & 1290 & $\mathrm{~m} 3$ & 36 & Univ. Utrecht & -10.4 & -2.9 \\
\hline Hipparion & primigenium & Nombrevilla & NOM & C.-Daroca & ESUU & 10.8 & 1290 & m3 & 39 & Univ. Utrecht & -10.5 & -2.9 \\
\hline Hipparion & primigenium & Nombrevilla & NOM & C.-Daroca & ESUU & 10.8 & 1290 & m3 & 41 & Univ. Utrecht & -10.5 & -3.2 \\
\hline Hipparion & primigenium & Nombrevilla & NOM & C.-Daroca & ESUU & 10.8 & 1290 & $\mathrm{~m} 3$ & 51 & Univ. Utrecht & -10.7 & -2.1 \\
\hline
\end{tabular}

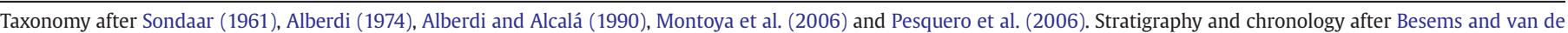

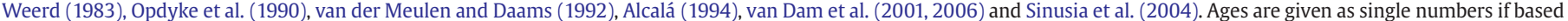
on magnetostratigraphy (using thicknesses to interpolate within chrons), or as ranges if based on biostratigraphy and/or lithostratigraphy unn.=unnumbered.

In this paper we study enamel $\delta^{18} \mathrm{O}$ compositions of a chronological series of Late Neogene (11-2 Ma) fossil horse teeth (Hipparion and Equus) from east Central Spain. Horses have a number of additional advantages for paleoclimate studies in comparison to other mammals. They have a wide geographic and temporal range, and are abundant as fossils. Because of their high water turnover rates the relation to $\delta^{18} \mathrm{O}_{\mathrm{H} 2 \mathrm{O}}$ is stronger compared to that in many other animals that obtain a large amount of water from leaves, which are enriched in ${ }^{18}$ O (Delgado Huertas et al., 1995; Hoppe et al., 2004a; Levin et al., 2006). In addition, the number of stable isotopes studies on fossil horse teeth from other parts of the world has increased significantly, resulting in a growing data base for comparison (Bryant et al., 1994; Sánchez-Chillón et al., 1994; Wang et al., 1994; Delgado Huertas et al., 1995; Bryant et al., 1996a, 1996b; Kohn et al., 1998; Sharp and Cerling, 1998; Feranec and MacFadden, 2000; Kohn et al., 2002; Passey et al., 2002; Hoppe et al., 2004a; Nelson, 2005).

Although enamel primarily consists of phosphate (apatite), we studied the (structural) carbonate of the enamel, which can reach up to $5 \%$ in mammal teeth (Rink and Schwarz, 1995). An advantage of measuring the carbonate is the additional availability of $\delta^{13} \mathrm{C}$ compositions, which provide information on diet and vegetation (e.g. Quade and Cerling, 1995; Cerling et al., 1997; Koch, 1998; Tipple and Pagani, 2007). $\delta^{13} \mathrm{C}$ in herbivore teeth mainly reflects the proportion of C4 (tropical grasses) and C3 plants (remaining, mostly temperate zone plants) in the diet. Because of an extremely efficient $\mathrm{CO}_{2}$ fixation, $\mathrm{C} 4$ plants have relatively high $\delta^{13} \mathrm{C}$ values. The average of $-12 \%$ o is closer to that of pre-industrial atmospheric $\mathrm{CO}_{2}(-6.5 \%)$, compared to C3 plants, which have a mean of $-26 \%$ (Smith and Epstein, 1971; Koch, 1998). The formation of tooth apatite in large herbivores is accompanied by a carbon isotopic fractionation of $~ 13 \%$ (Koch, 1998), so that average values of -13 and $+1 \%$ o characterize the apatite of pure C3 and C4 consumers, respectively. Differences within the C3 isotopic spectrum in apatite (from -20 to -7\%) usually reflect gradients from closed canopy to open, more water-stressed environments (Farquhar et al., 1989; Van der Merwe and Medina, 1991; Ehleringer and Monson, 1993; Passey et al., 2002).

\section{Intra-tooth isotopic variability}

High-crowned (hypsodont) teeth grow at the crown base, and therefore the crown top is the oldest part (Fig. 1). Molars and preõmolars of the modern horse Equus caballus reach their full height ( 40-60 mm) in early life within 1-1.5 years (Hoppe et al., 2004b).
Subsequent growth compensates wear at the tooth crown and is very slow (maximally a few $\mathrm{mm}$ per year). Although seasonal variability in the isotopic composition of the drinking water is recorded in the dentition, the signal is both damped and delayed. Damping, expressed by the damping factor (which we call $d$ ), is defined here as the difference between the amplitude in the drinking water $\left(\delta^{18} \mathrm{O}_{\mathrm{H} 2 \mathrm{O}}\right)$ and the measured amplitude in the tooth $\left(\delta^{18} \mathrm{O}_{\mathrm{PO} 4}\right)$, relative to the amplitude in the drinking water (Passey and Cerling, 2002; Kohn, 2004).

The damping factor is dependent on at least four factors. First, a damping of $25( \pm 5) \%$ of the oxygen isotopic signal is produced by a $\sim 25 \%$ contribution of atmospheric oxygen which is fixed in the animal with a $\delta^{18} \mathrm{O}$ of $15 \%$ (Kohn and Cerling, 2002). This damping is reflected by a slope of $\sim 0.75$ instead of the expected 1.0 in $\delta^{18} \mathrm{OPO}_{\mathrm{PO}}$ $\delta^{18} \mathrm{O}_{\mathrm{H} 20}$ equations ( 0.71 in horses, see below). Second, the residence time of oxygen in the body, which is in the order of 10-20 days, causes 10\% damping (Kohn et al., 1998; 2002; Kohn and Cerling, 2002), Third, damping occurs because the mineralization process of the enamel from a initial soft matrix to its final dense form takes a certain amount of time. This maturation interval is not known for horses, but Kohn (2004) estimated it to be $\sim 5$ months, based on general size and data from other large herbivores. This duration translates to a distance of $\sim 2-2.5 \mathrm{~cm}$ along the lateral side of the growing tooth (Passey and Cerling, 2002; Kohn, 2004). Fourth, significant time averaging occurs by collecting samples perpendicularly to the lateral side thereby crossing oblique mineralization fronts. If samples include the full enamel depth $(\sim 1 \mathrm{~mm})$, time averaging is in the order of three months, which corresponds to a distance of $\sim 1.5 \mathrm{~cm}$ along the crown (Hoppe et al., 2004b).

In Fig. 2 we show what would happen to an environmental signal as a consequence of these four types of successive and partially overlapping sorts of damping. The environmental signal in Fig. 2 is based on real data, namely (unweighted) daily temperature data for the town of Teruel, Central Spain during the years 1996-2001. (No daily $\delta^{18} \mathrm{O}_{\mathrm{H} 20}$ data are available for the Iberian Peninsula.). The calculation of the maturation and sampling effects is based on the model of Passey and Cerling (2002). In this model it is assumed that the isotopic composition at a certain point is consisting of contributions from an initial enamel matrix (representing $25 \%$ of the final material) and from portions of material subsequently added along oblique apposition fronts during further enamel maturation (the remaining 75\%). As mentioned above, the amplitude of the horse body water will first be damped by $\sim 25 \%$ due to the mixing 
Summary of $\delta^{18} \mathrm{O}$ and $\delta^{13} \mathrm{C}$ measurements and interpretations for twelve isotopic profiles across the enamel of horse teeth from localities in east Central and East Spain

\begin{tabular}{|c|c|c|c|c|c|c|c|c|c|c|c|c|c|c|c|}
\hline Locality & Code & Basin & $\begin{array}{l}\text { Altitude } \\
(\mathrm{m})\end{array}$ & MN unit & Local zone & $\begin{array}{l}\text { Age } \\
\text { range } \\
\text { (Ma) }\end{array}$ & $\begin{array}{l}\text { Specimen } \\
\text { number }\end{array}$ & Element & $\begin{array}{l}\text { Number } \\
\text { of sub- } \\
\text { samples }\end{array}$ & $\begin{array}{l}\delta^{13} \mathrm{C}(\%) \\
\text { (VPDB) } \\
\text { mean }\end{array}$ & $\begin{array}{l}\delta^{13} \mathrm{C}(\%) \\
\text { (VPDB) } \\
\text { midpoint }\end{array}$ & $\begin{array}{l}\delta^{13} \mathrm{C}(\%) \\
\text { (VPDB) } \\
\text { min. }\end{array}$ & $\begin{array}{l}\delta^{13} \mathrm{C}(\%) \\
\text { (VPDB) } \\
\text { corr. min. } \\
(d=0.5)\end{array}$ & $\begin{array}{l}\delta^{13} \mathrm{C}(\%) \\
(\mathrm{VPDB}) \\
\text { max. }\end{array}$ & $\begin{array}{l}\delta^{13} \mathrm{C}(\%) \\
\text { (VPDB) } \\
\text { corr. max. } \\
(d=0.5)\end{array}$ \\
\hline $\begin{array}{l}\text { La Puebla } \\
\text { de Valverde }\end{array}$ & PVAL & Teruel & 1118 & 17 & Not defined & 2.1 & Not assigned & M3 & 10 & -11.30 & -11.26 & -11.57 & -11.88 & -10.95 & -10.64 \\
\hline Orrios & OR & Teruel & 900 & 14 & Trilophomys & 4.4 & 212 & $\mathrm{~m} 3$ & 18 & -11.60 & -11.60 & -11.86 & -12.12 & -11.34 & -11.07 \\
\hline La Calera & LCA & Teruel & 900 & 14 & Trilophomys? & $4.8-3.8$ & 81 & M3 & 10 & -11.42 & -11.27 & -11.71 & -12.15 & -10.82 & -10.38 \\
\hline $\begin{array}{l}\text { Venta del } \\
\text { Moro }\end{array}$ & VM & Cabriel & 725 & 13 & M3 & 6.0 & Not assigned & M3 & 18 & -10.31 & -10.44 & -11.08 & -11.71 & -9.81 & -9.18 \\
\hline El Arquillo & ARQ & Teruel & 900 & 13 & M2 & $6.4-6.2$ & Not assigned & M3 & 18 & -11.22 & -11.20 & -11.49 & -11.79 & -10.91 & -10.61 \\
\hline $\begin{array}{l}\text { Concud } \\
\text { Cerro de } \\
\text { La Garita }\end{array}$ & CC & Teruel & 900 & 12 & $\mathrm{~L}$ & $7.2-7.1$ & 277 & M3 & 18 & -10.17 & -10.23 & -10.73 & -11.24 & -9.72 & -9.21 \\
\hline $\begin{array}{l}\text { Puente } \\
\text { Minero }\end{array}$ & $\mathrm{PM}$ & Teruel & 900 & 11 & K & $8.7-8.3$ & 212 & $\mathrm{~m} 3$ & 25 & -10.63 & -10.64 & -11.12 & -11.59 & -10.16 & -9.68 \\
\hline La Roma 2 & R2 & Teruel & 900 & 10 & $\mathrm{~J} 3$ & $9.3-9.2$ & 2065 & M3 & 19 & -9.90 & -9.97 & -10.63 & -11.28 & -9.31 & -8.66 \\
\hline $\begin{array}{l}\text { Masía del } \\
\text { Barbo 2A }\end{array}$ & MBA & Teruel & 900 & 10 & $\mathrm{~J} 1$ & 9.7 & 52 & $\mathrm{~m} 3$ & 16 & -10.34 & -10.35 & -11.04 & -11.73 & -9.66 & -8.96 \\
\hline $\begin{array}{l}\text { Pedregueras } \\
2 \mathrm{~A}\end{array}$ & PED2A & Cal.-Daroca & 797 & 9 & I & 10.0 & Not assigned & $\mathrm{M} 1 / 2$ & 21 & -10.35 & -10.36 & -10.67 & -10.98 & -10.05 & -9.75 \\
\hline Nombrevilla & NOM & Cal.-Daroca & 736 & 9 & $\mathrm{H}$ & 10.8 & 1290 & m3 & 13 & -10.53 & -10.81 & -11.37 & -11.93 & -10.25 & -9.69 \\
\hline Nombrevilla & NOM & Cal.-Daroca & 736 & 9 & $\mathrm{H}$ & 10.8 & 1188 & M3 & 24 & -10.29 & -10.18 & -10.71 & -11.25 & -9.64 & -9.11 \\
\hline Locality & $\begin{array}{l}\text { Specimen } \\
\text { number }\end{array}$ & $\begin{array}{l}\delta^{13} \mathrm{C}(\%) \\
\text { (VPDB) } \\
\text { range }\end{array}$ & $\begin{array}{l}\delta^{13} \mathrm{C}(\%) \\
\text { (VPDB) } \\
\text { corr. } \\
\text { range } \\
(d=0.5)\end{array}$ & $\begin{array}{l}\delta^{13} \mathrm{C}- \\
\delta^{18} \mathrm{O}(\%) \\
\text { Pearson's } r\end{array}$ & $\begin{array}{l}\delta^{18} \mathrm{O}_{\mathrm{CO}} \\
(\%)(\mathrm{VPDB}) \\
\text { mean }\end{array}$ & $\begin{array}{l}\delta^{18} \mathrm{O}_{\mathrm{CO} 3} \\
\text { (\%) } \\
\text { (VPDB) } \\
\text { midpoint }\end{array}$ & $\begin{array}{l}\delta^{18} \mathrm{O}_{\text {соз }} \\
\text { (\%) } \\
\text { (VSMOW) } \\
\text { midpoint } \\
\text { (Eq. (1)) }\end{array}$ & $\begin{array}{l}\delta^{18} \mathrm{O}_{\mathrm{PO} 4} \\
(\%) \\
\text { (VSMOW) } \\
\text { mean } \\
\text { (Eq. (2)) }\end{array}$ & $\begin{array}{l}\delta^{18} \mathrm{O}_{\mathrm{PO} 4} \\
(\% \circ) \\
\text { (VSMOW) } \\
\text { midpoint }\end{array}$ & $\begin{array}{l}\delta^{18} \mathrm{O}_{\mathrm{PO} 4} \\
(\%) \\
\text { (VSMOW) } \\
\text { min. } \\
\text { (Eq. (2)) }\end{array}$ & $\begin{array}{l}\delta^{18} \mathrm{O}_{\mathrm{PO} 4} \\
(\%) \\
\text { (VSMOW) } \\
\max . \\
\text { (Eq. (2)) }\end{array}$ & $\begin{array}{l}\delta^{18} \mathrm{O}_{\mathrm{H} 2 \mathrm{O}} \\
(\%) \\
\text { (VSMOW) } \\
\text { midpoint } \\
\text { (Eq. (4)) }\end{array}$ & $\begin{array}{l}\delta^{18} \mathrm{O}_{\mathrm{H} 2 \mathrm{O}} \\
(\%) \\
(\mathrm{VSMOW}) \\
\text { mean } \\
\text { (Eq. (4)) }\end{array}$ & $\begin{array}{l}\delta^{18} \mathrm{O}_{\mathrm{H} 2 \mathrm{O}} \\
(\% \circ) \\
\text { (VSMOW) } \\
\text { correct. } \\
\text { min. } \\
(c=0.6) \\
\end{array}$ & $\begin{array}{l}\delta^{18} \mathrm{O}_{\mathrm{H} 2 \mathrm{O}} \\
(\%) \\
\text { (VSMOW) } \\
\text { correct. } \\
\text { min. } \\
(c=0.75)\end{array}$ \\
\hline $\begin{array}{l}\text { La Puebla de } \\
\text { Valverde }\end{array}$ & Not assigned & 0.62 & 1.25 & 0.23 & -4.31 & -4.55 & 26.17 & 17.39 & 17.15 & 16.29 & 18.01 & -7.64 & -7.31 & -9.76 & -10.24 \\
\hline Orrios & 212 & 0.52 & 1.05 & 0.38 & -3.80 & & & 17.90 & & 17.27 & 18.49 & & & & \\
\hline La Calera & 81 & 0.88 & 1.77 & -0.69 & -3.06 & -3.24 & 27.52 & 18.66 & 18.47 & 17.58 & 19.35 & -5.80 & -5.53 & -7.96 & -8.46 \\
\hline $\begin{array}{l}\text { Venta } \\
\text { del Moro }\end{array}$ & Not assigned & 1.26 & 2.52 & 0.76 & -1.94 & -2.18 & 28.61 & 19.78 & 19.54 & 18.42 & 20.65 & -4.30 & -3.95 & -7.03 & -7.66 \\
\hline
\end{tabular}

del Moro 


\begin{tabular}{|c|c|c|c|c|c|c|c|c|c|c|c|c|c|c|c|}
\hline El Arquillo & Not assigned & 0.59 & 1.18 & 0.11 & -2.97 & -3.08 & 27.68 & 18.74 & 18.63 & 17.34 & 19.91 & -5.57 & -5.41 & -8.72 & -9.44 \\
\hline $\begin{array}{l}\text { Concud Cerro de } \\
\text { La Garita }\end{array}$ & 277 & 1.01 & 2.03 & -0.79 & -3.16 & -3.17 & 27.59 & 18.55 & 18.54 & 17.46 & 19.62 & -5.70 & -5.68 & -8.34 & -8.95 \\
\hline $\begin{array}{l}\text { Puente } \\
\text { Minero }\end{array}$ & 212 & 0.96 & 1.92 & 0.15 & -3.24 & -3.19 & 27.58 & 18.47 & 18.52 & 17.47 & 19.58 & -5.71 & -5.79 & -8.29 & -8.88 \\
\hline La Roma 2 & 2065 & 1.31 & 2.62 & 0.80 & -1.99 & -1.95 & 28.85 & 19.73 & 19.77 & 18.27 & 21.27 & -3.96 & -4.03 & -7.64 & -8.49 \\
\hline $\begin{array}{l}\text { Masía del } \\
\text { Barbo 2A }\end{array}$ & 52 & 1.38 & 2.77 & -0.48 & -0.14 & & & 21.61 & & 20.68 & 22.73 & & & & \\
\hline $\begin{array}{l}\text { Pedregueras } \\
2 \mathrm{~A}\end{array}$ & Not assigned & 0.61 & 1.23 & 0.46 & -1.24 & -1.16 & 29.66 & 20.49 & 20.57 & 19.11 & 22.03 & -2.85 & -2.95 & -6.42 & -7.24 \\
\hline Nombrevilla & 1290 & 1.12 & 2.24 & -0.25 & -2.69 & & & 19.02 & & 18.54 & 19.66 & & & & \\
\hline Nombrevilla & 1188 & 1.07 & 2.14 & -0.25 & -2.39 & -2.42 & 28.36 & 19.33 & 19.30 & 18.27 & 20.32 & -4.63 & -4.59 & -7.14 & -7.72 \\
\hline Locality & $\begin{array}{l}\text { Specimen } \\
\text { number }\end{array}$ & $\begin{array}{l}\delta^{18} \mathrm{O}_{\mathrm{H} 2 \mathrm{O}}(\%) \\
\text { (VSMOW) } \\
\text { corrected } \\
\text { max. } \\
(d=0.6)\end{array}$ & $\begin{array}{l}\delta^{18} \mathrm{O}_{\mathrm{H} 2 \mathrm{O}}(\%) \\
\text { (VSMOW) } \\
\text { corrected } \\
\text { max. } \\
(d=0.75)\end{array}$ & $\begin{array}{l}\delta^{18} \mathrm{O}_{\mathrm{H} 2 \mathrm{O}}(\%) \\
\text { (VSMOW) } \\
\text { corrected } \\
\text { range } \\
(d=0.6)\end{array}$ & $\begin{array}{l}\delta^{18} \mathrm{O}_{\mathrm{H} 2 \mathrm{O}}(\%) \\
\text { (VSMOW) } \\
\text { corrected } \\
\text { range } \\
(d=0.75)\end{array}$ & $\begin{array}{l}\delta^{18} \mathrm{O}_{\mathrm{H} 2 \mathrm{O}}(\%) \\
\text { (VSMOW) } \\
\text { sea water } \\
\text { (Lear et al., } \\
2000 \text { ) }\end{array}$ & $\begin{array}{l}\text { Annual } T\left({ }^{\circ} \mathrm{C}\right) \\
\text { from mean } \\
\text { subsamples } \\
\text { (Eq. (9)) }\end{array}$ & $\begin{array}{l}\text { Annual } T\left({ }^{\circ} \mathrm{C}\right) \\
\text { from mean } \\
\delta^{18} \mathrm{O}_{\mathrm{H} 2 \mathrm{O}} \\
(\text { Eq. (7)) }\end{array}$ & $\begin{array}{l}\text { Annual } T\left({ }^{\circ} \mathrm{C}\right) \\
\text { from } \\
\text { midpoint } \\
\delta^{18} \mathrm{O}_{\mathrm{H} 2 \mathrm{O}} \\
\text { (Eq. (7)) }\end{array}$ & $\begin{array}{l}T \text { min. }\left({ }^{\circ} \mathrm{C}\right) \\
\text { from min. } \\
\delta^{18} \mathrm{O}_{\mathrm{H} 2 \mathrm{O}} \\
(d=0.6) \\
\text { (Eq. (9)) }\end{array}$ & $\begin{array}{l}T \text { min. }\left({ }^{\circ} \mathrm{C}\right) \\
\text { from min. } \\
\delta^{18} \mathrm{O}_{\mathrm{H} 2 \mathrm{O}} \\
(d=0.75) \\
\text { (Eq. (9)) }\end{array}$ & $\begin{array}{l}T \text { max. }\left({ }^{\circ} \mathrm{C}\right) \\
\text { from max. } \\
\delta^{18} \mathrm{O}_{\mathrm{H} 2 \mathrm{O}} \\
(d=0.6) \\
\text { (Eq. (9)) }\end{array}$ & $\begin{array}{l}T \text { max. }\left({ }^{\circ} \mathrm{C}\right) \\
\text { from max. } \\
\delta^{18} \mathrm{O}_{\mathrm{H} 2 \mathrm{O}} \\
(d=0.75) \\
\text { (Eq. (9)) }\end{array}$ & $\begin{array}{l}T \text { range }\left({ }^{\circ} \mathrm{C}\right) \\
\text { from range } \\
\delta^{18} \mathrm{O}_{\mathrm{H} 2 \mathrm{O}} \\
(d=0.6)\end{array}$ & $\begin{array}{l}T \text { range }\left({ }^{\circ} \mathrm{C}\right) \\
\text { from range } \\
\delta^{18} \mathrm{O}_{\mathrm{H} 2 \mathrm{O}} \\
(d=0.75)\end{array}$ \\
\hline $\begin{array}{l}\text { La Puebla } \\
\text { de Valverde }\end{array}$ & Not assigned & -5.53 & -5.04 & 4.23 & 5.20 & 0.10 & 8.0 & 9.6 & 8.9 & 1.5 & 0.3 & 12.6 & 13.9 & 11.1 & 13.7 \\
\hline Orrios & 212 & & & & & -0.60 & 11.7 & 12.5 & & & & & & & \\
\hline La Calera & 81 & -3.63 & -3.13 & 4.34 & 5.34 & -0.60 & 14.5 & 14.6 & 14.1 & 8.1 & 6.8 & 19.5 & 20.8 & 11.4 & 14.0 \\
\hline $\begin{array}{l}\text { Venta del } \\
\text { Moro }\end{array}$ & Not assigned & -1.56 & -0.93 & 5.47 & 6.73 & -0.50 & 13.0 & 12.2 & 11.7 & 10.3 & 8.6 & 24.6 & 26.3 & 14.4 & 17.7 \\
\hline El Arquillo & Not assigned & -2.42 & -1.70 & 6.30 & 7.75 & -0.40 & 14.2 & 14.4 & 14.1 & 5.6 & 3.7 & 22.1 & 24.0 & 16.5 & 20.3 \\
\hline $\begin{array}{l}\text { Concud } \\
\text { Cerro de } \\
\text { La Garita }\end{array}$ & 277 & -3.05 & -2.44 & 5.29 & 6.51 & -0.25 & 13.1 & 13.6 & 13.6 & 6.2 & 4.6 & 20.1 & 21.7 & 13.9 & 17.1 \\
\hline $\begin{array}{l}\text { Puente } \\
\text { Minero }\end{array}$ & 212 & -3.14 & -2.54 & 5.16 & 6.34 & -0.25 & 12.9 & 13.4 & 13.5 & 6.3 & 4.7 & 19.8 & 21.4 & 13.5 & 16.7 \\
\hline $\begin{array}{l}\text { La Roma } 2 \\
\text { Masía del Barbo 2A }\end{array}$ & $\begin{array}{l}2065 \\
52\end{array}$ & -0.29 & 0.56 & 7.35 & 9.04 & $\begin{array}{l}-0.25 \\
-0.35\end{array}$ & 17.5 & 17.0 & 17.1 & 8.0 & 5.8 & 27.3 & 29.5 & 19.3 & 23.8 \\
\hline $\begin{array}{l}\text { Pedregueras 2A } \\
\text { Nombrevilla }\end{array}$ & $\begin{array}{l}\text { Not assigned } \\
1290\end{array}$ & 0.73 & 1.55 & 7.15 & 8.80 & $\begin{array}{l}-0.40 \\
-0.30\end{array}$ & $\begin{array}{l}\sim 20^{\mathrm{a}} \\
15.0\end{array}$ & $\begin{array}{l}\sim 19^{\mathrm{a}} \\
15.1\end{array}$ & $\sim 19^{\mathrm{a}}$ & 11.6 & 9.4 & $29-30^{\mathrm{a}}$ & $29-33^{\mathrm{a}}$ & $17-19^{\mathrm{a}}$ & $19-23^{\mathrm{a}}$ \\
\hline $\begin{array}{l}\text { Nombrevilla } \\
\text { Nombrevilla }\end{array}$ & $\begin{array}{l}1290 \\
1188\end{array}$ & -2.12 & -1.54 & 5.02 & 6.18 & $\begin{array}{l}-0.30 \\
-0.30\end{array}$ & 16.2 & 15.9 & 15.8 & 9.4 & 7.9 & 22.6 & 24.1 & 13.2 & 16.2 \\
\hline
\end{tabular}

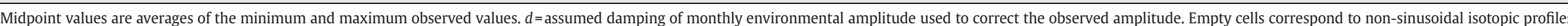
Midpoint values are averages of the minimum and maximum observed values. $d=$ assumed damping of monthy 1 (Nombrevilla, Masía del Barbo and Orrios). Taxonomic and stratigraphic/chronological references: see Table 1.
(.

a Uncertainty because of presumed evaporative effects. 
with atmospheric $\mathrm{O}_{2}$. Simulations of the reservoir and maturation effects on the example data result in additional damping steps of 36 and $41 \%$, respectively (four-year average). The resulting cumulative damping amounts to $73 \%$. The associated time lag between the environmental and isotopic signal is three months. The largest uncertainties are associated with the maturation effect. If enamel maturation time is assumed to last only three instead of five months, damping due to maturation would drop from 41 to $24 \%$ and the time lag between the environmental and isotopic signal would be one month shorter.

Sampling the complete enamel depth $(\sim 1 \mathrm{~mm}$, corresponding to 3 months) by a theoretical sample of which the height corresponds to only one day (i.e. the same time resolution as the original signal), increases total damping from 73 to $77 \%$. The effect of sampling depth on the measured amplitude is small, because significant time averaging has already been produced by the reservoir and maturation effects. Note that the calculated damping corresponds to a daily time scale $\left(d_{d}=0.77\right.$, which is applied to an annual environmental amplitude $A_{d}$ based on daily data in Fig. 2). If monthly data are used, damping will be less $\left(d_{m}=0.63\right.$, which is applied to an annual environmental amplitude $A_{m}$ based on monthly data in Fig. 2). The additional signal delay of one and a half month after sampling shown in Fig. 2 reflects the choice of the chronological reference, which is chosen to be at the surface of the tooth.

In addition to these internal damping factors, external damping may occur because of mixing within drinking water source bodies. This buffering effect will be more profound for larger water bodies (see Kohn and Cerling, 2002). A second type of external buffering occurs when animals migrate, and track the isotopic environment. On the other hand, the measured seasonal amplitude may become larger when a broader range of $\delta^{18} \mathrm{O}$ values is traversed (Bryant et al., 1996b; Hoppe, 2006).

\section{Material and methods}

The studied equid teeth originate from various Late Miocene and Pliocene fossil localities in east Central Spain spanning the interval between 11 and $2 \mathrm{Ma}$ (Tables 1 and 2). The majority of the material was collected in the Teruel Basin (Masía del Barbo 2A/2B, Alto de Marinezquita, La Roma 2, Puente Minero, Concud Cerro de la Garita, Los Mansuetos, El Arquillo, Las Casiones, Milagros, La Gloria 4, La Calera, Orrios, La Puebla de Valverde). Additional material originates from the Calatayud-Daroca Basin situated $\sim 100 \mathrm{~km}$ north (Nombrevilla, Pedregueras 2A) and the Cabriel Basin situated $\sim 150 \mathrm{~km}$ south (Venta del Moro) of the Teruel Basin localities. For information on the localities and their ages is referred to Besems and Van de Weerd (1983), Opdyke et al. (1990), van der Meulen and Daams (1992), Alcalá (1994), van Dam et al. (2001, 2006) and Sinusia et al. (2004). Taxonomic information can be found in various studies, such as those of Sondaar (1961), Alberdi (1974), Alberdi and Alcalá (1990), Montoya et al. (2006) and Pesquero et al. (2006).

Twelve equid teeth were sampled in detail from the occlusal surface down to the crown base in order to study intra-tooth variation (Table 2). Judged from wear and general shape, eight of these were from relatively young individuals. The number of sub-samples per tooth varied between 10 and 26 with an average of 17.5. Because the isotopic amplitude is strongly time-averaged, no effort was taken to standardize the number of sub-samples per tooth. A second set of eleven teeth were sampled by one or two sub-samples in order to check between-specimen and between-locality variation (Table 1). A third set of unpublished isotopic compositions from twenty teeth from the Teruel basin were kindly provided to us by Dr. Thure Cerling (University of Utah). All teeth in our study except for one (an Equus tooth from La Puebla de Valverde) belong to the extinct genus Hipparion. During the Late Pliocene Equus replaces Hipparion in Europe and elsewhere.
The majority of the studied teeth are third molars (M3/m3). These were preferred for isotopic analysis because other elements such as second and particularly first molars could be affected by the mother's body water composition via milk (Bryant et al., 1994). A number of other studies suggest that there is no significant lactation effect on $\delta^{18}$ O, however (Kohn et al., 1998; Gadbury et al., 2000; Nelson, 2005). Zazzo et al. (2000) inferred $0.6-0.7 \%$ o decrease in $\delta^{13} \mathrm{C}$ (due to milk fat) for fossil bovids.

Tooth surfaces were cleaned mechanically with a small lowspeed circle saw, which was also used to collect enamel powder. Samples were taken perpendicularly to the lateral side of the crown, producing (series of) small furrows with a height of $\sim 0,8 \mathrm{~mm}$ (i.e. in the direction of growth), a maximal depth of $\sim 1 \mathrm{~mm}$ and a length of $\sim 5 \mathrm{~mm}$. The samples were soaked with $2 \%$ sodium hypochlorite $(\mathrm{NaOCl})$ to oxydize organic matter, rinsed three times with distilled water, and subsequently soaked with $0.1 \mathrm{M}$ acetic acid to remove diagenetic carbonates and again rinsed three times (Lee-Thorp and van der Merwe, 1987; Koch et al., 1997). The samples were transferred to an automated carbonate preparation unit (IsoCarb), after which the isotopes were measured on an isotope ration mass spectrometer (VG SIRA 24). Initial values are reported relative to the Vienna Pee Dee Belemnite (VPDB) in standard $\delta$ notation; calibration is achieved through analyses of National Bureau of Standards 19 reference material. Precision of the $\delta^{18} \mathrm{O}$ and $\delta^{13} \mathrm{C}$ analyses was better than $0.1 \%$ and $0.05 \%$ 。 respectively. The University of Utah samples were measured on a Finnegan MAT 252 mass spectrometer. Sample pretreatment was similar as described above, except for using $3 \% \mathrm{H}_{2} \mathrm{O}_{2}$ instead of $2 \%$ sodium hypochlorite $(\mathrm{NaOCl})$ to remove organic matter (Cerling, pers. comm.).

In order to estimate $\delta^{18} \mathrm{O}_{\mathrm{H} 2 \mathrm{O}}, \delta^{18} \mathrm{O}_{\mathrm{CO} 3}$ values were first converted from the VPDB to the VSMOW standard scale (Friedman and O'Neil, 1977):

$\delta^{18} \mathrm{O}_{\mathrm{CO} 3}(\mathrm{VSMOW})=1.03086^{*} \delta^{18} \mathrm{O}_{\mathrm{CO} 3}(\mathrm{VPDB})+30.86$

and then to $\delta^{18} \mathrm{O}_{\mathrm{PO} 4}-$ equivalent values (Iacumin et al., 1996):

$\delta^{18} \mathrm{O}_{\mathrm{PO} 4}(\mathrm{VSMOW})=\delta^{18} \mathrm{O}_{\mathrm{CO} 3}(\mathrm{VSMOW}) * 0.98-8.5$

We use the isotopic scale for horses compiled by Delgado Huertas et al. (1995), which is partly based on Bryant et al. (1994) and Sánchez-Chillón et al. (1994). This scale relates $\delta^{18} \mathrm{O}_{\mathrm{PO} 4}$ from recent horse bone and teeth to local $\delta^{18} \mathrm{O}_{\mathrm{H} 2 \mathrm{O}}$ from different places across the world. The scale is based on the following least squares (LS) regression:

$$
\begin{aligned}
& \delta^{18} \mathrm{O}_{\mathrm{PO} 4}(\mathrm{VSMOW})=0.713 * \delta^{18} \mathrm{O}_{\mathrm{H} 2 \mathrm{O}}(\mathrm{VSMOW})+22.605 \\
& \left(R^{2}=0.77\right)
\end{aligned}
$$

with standard errors $(\sigma)$ for the slope and intercept of 0.08 and 0.60 , respectively. In order to predict $\delta^{18} \mathrm{O}_{\mathrm{H} 2 \mathrm{O}}$ we used the inversed form of Eq. (3):

$\delta^{18} \mathrm{O}_{\mathrm{H} 2 \mathrm{O}}(\mathrm{VSMOW})=1.403 * \delta^{18} \mathrm{O}_{\mathrm{PO} 4}(\mathrm{VSMOW})-31.704$

\section{Results}

4.1. Stable oxygen isotope compositions and temperatures in present-day Iberia

Fig. 3a shows seasonal curves of $\delta^{18} \mathrm{O}_{\mathrm{H} 2 \mathrm{O}}$ (IEAE, 2005) and temperature for the Iberian Peninsula (see caption). The graphs show the distinct within-year relation between $\delta^{18} \mathrm{O}_{\mathrm{H} 2 \mathrm{O}}$ and temperature as well the expected geographical trends towards more depleted values in the north and the east. This geographical patterns is caused by the combined latitudinal, continental and 

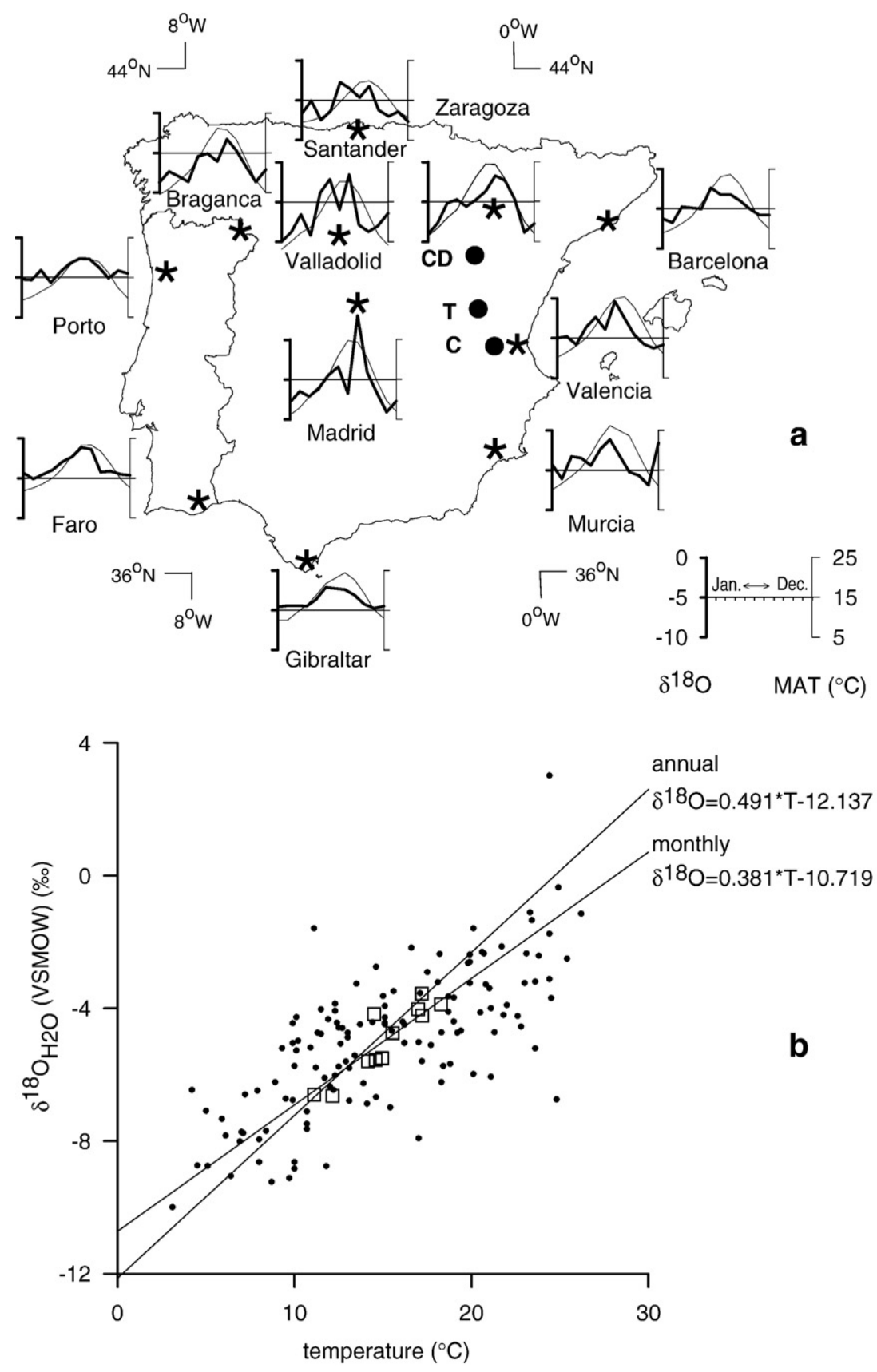

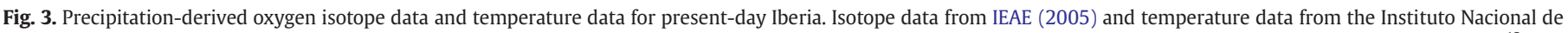

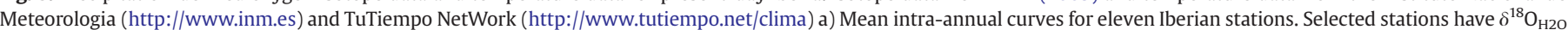

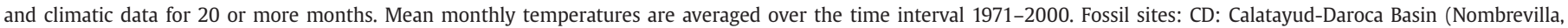

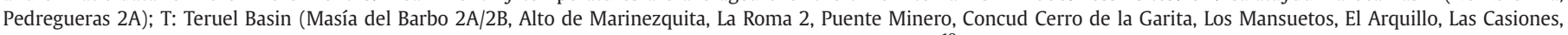

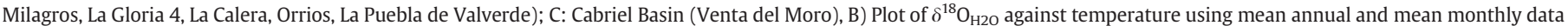
with Reduced Major Axis (RMA) regression lines. Squares: annual values, dots: monthly values.

altitudinal effects on the dominantly Atlantic air masses, which preferentially loose ${ }^{18} \mathrm{O}$ while traversing Spain. Values along the coastal regions of E Spain are somewhat higher than expected, which results both from the occurrence of mild winters as well as the additional contribution of the nearby Mediterranean water sources (Vallejos et al., 1997). One distinct outlier of $+3.0 \%$ o (Madrid. August 2001) either could correspond to an erroneous measurement or to a short interval of abnormally strong evaporation and/or precipitation recycling.

Fig. $3 \mathrm{~b}$ shows the direct relation between $\delta^{18} \mathrm{O}_{\mathrm{H} 2 \mathrm{O}}$ and temperature for annual and monthly means. Stepwise linear regression (LS) of mean annual (MA) $\delta^{18} \mathrm{O}_{\mathrm{H} 2 \mathrm{O}}$ on mean annual temperature (MAT), pre- cipitation, latitude, longitude and altitude results in a significant fit with temperature only:

$$
\begin{aligned}
& \mathrm{MA} \delta^{18} \mathrm{O}_{\mathrm{H} 2 \mathrm{O}}=0.447 * \mathrm{MAT}-11.717 \\
& \left(R^{2}=0.83\right)
\end{aligned}
$$

with an associated $\sigma$ of 0.07 for the slope. Reduced Major Axis (RMA) is probably a more appropriate method than the more commonly used LS because it adresses uncertainties in both $\delta^{18} \mathrm{O}_{\mathrm{H} 2 \mathrm{O}}$ and temperature: mean (monthly) temperatures are only a crude approximation of the temperatures during the precipitation events themselves, to which the $\delta^{18} \mathrm{O}_{\mathrm{H} 2 \mathrm{O}}$ measurements would actually 
apply (Kohn and Welker, 2005). RMA results in an equation which is nevertheless very similar:

$\mathrm{MA} \delta^{18} \mathrm{O}_{\mathrm{H} 2 \mathrm{O}}=0.491 * \mathrm{MAT}-12.389$

with $\sigma=0.06$ for the slope. The spatial (mean annual) slope of $0.45-$ 0.5 for Iberia is slightly lower than Rozanski et al.'s (1992) value of 0.59 as inferred for Central-North Europe $\left(40-60^{\circ} \mathrm{N}\right)$. This difference is in agreement with the expected poleward increase of slope values. The inversed form of Eq. (6) is as follows:

MAT $=2.037 * \mathrm{MA} \delta^{18} \mathrm{O}_{\mathrm{H} 2 \mathrm{O}}+25.232$

As expected, the RMA slope estimate using mean monthly (MM) data is lower, namely $0.38(\sigma=0.08)$ :

$\mathrm{MM} \delta^{18} \mathrm{O}_{\mathrm{H} 2 \mathrm{O}}=0.381 * \mathrm{MMT}-10.719$

$\left(R^{2}=0.56\right)$

or:

$\mathrm{MMT}=2.626^{*} \mathrm{MM} \delta^{18} \mathrm{O}_{\mathrm{H} 2 \mathrm{O}}+28.151$

The LS slope estimate is 0.27 , which again is slightly lower than the European values of 0.31 inferred by Rozanski et al. (1993) using LS estimation for monthly data. Interestingly, stepwise LS regression of the Iberian (monthly) values on monthly values of temperature and precipitation, latitude, longitude and altitude results in a best significant fit including not only mean monthly temperature but also altitude (in $\mathrm{km}$ ):

$\mathrm{MM} \delta^{18} \mathrm{O}_{\mathrm{H} 2 \mathrm{O}}=0.249 * \mathrm{MMT}-1.589 *$ ALT -8.345

$\left(R^{2}=0.56\right)$

with $\sigma=0.024,0.459,0.423$ for MMT, ALT and the constant, respectively. The altitudinal coefficient of $-1.6 \% / \mathrm{km}$ is at the lower end of observed values $(-1.5$ to $-5 \% / \mathrm{km}$, Siegenthaler and Oeschger, 1980; Yurtsever and Gat, 1981) but is underestimated in Eq. (10) because part of the altitude- $\delta^{18} \mathrm{O}_{\mathrm{H} 2 \mathrm{O}}$ effect is explained by temperature. A LS regression on altitude alone results in a coefficient of $-3.1 \%$ / $\mathrm{km}$. This value is very close to the value of $-3.4 \% / \mathrm{km}$ estimated across the Sierra de Gador, SE Spain (Vallejos et al., 1997). The LS slope of 0.25-0.27 for MMT is much lower than the spatial, mean annual LS slope of 0.45 (Eq. (5)). The RMA-based slopes are not so different ( 0.38 and 0.49 ), especially when the large uncertainties around the means are considered. RMA-estimated slopes for the individual stations of Zaragoza and Valencia (180 and $140 \mathrm{~km}$ from Teruel, respectively, Fig. 3a), have intermediate values of 0.45 and 0.40 (monthly data for the years 2000 and 2001). The LSbased slope estimates for these two stations are much lower: 0.30 and 0.20 .

In addition to spatial slopes, Rozanski et al. $(1992,1993)$ also calculated interannual (temporal) slopes for a number of European stations that have complete records spanning various decades. They found a mean slope value of 0.53 . This value is close to the European spatial slope of 0.59 . Unfortunately, such long ( $>2$ decades) and complete records are not available for the Iberian Peninsula.

\subsection{Late Neogene $\delta^{18} \mathrm{O}$ and temperature trends}

The stable isotope measurements of the fossil teeth are shown in Tables 1,2 and Figs. 4 and 5. The $\delta^{18} \mathrm{O}(\mathrm{VPDB})$ record shows a general decrease from mean values between -2 and $0 \%$ during the early Late Miocene (11-9 Ma) to values around $-4 \%$ o during the Late Pliocene (2 Ma). In order to interpret these temporal trends correctly, the effect of the geographic and climatologic separation of the localities should be taken into account. The bulk of the material originates from the
Teruel Basin, for which detailed modern climatic data exist (the town of Teruel). Unfortunately, only very sparse climate data exist for the town of Daroca (with the nearby localities Nombrevilla and Pedregueras 2A, Daroca-Calatayud Basin), hampering a quantitative meteorological comparison with Teruel (100 km south). Nevertheless, temperatures in the two areas can be assumed to be very similar, given their comparable topographic configuration in the same SSW-NNE trending depression in the Iberian Chain. The single other locality outside the Teruel Basin,Venta del Moro (VM), is situated close to the Mediterranean Sea (Valencia). Its $\delta^{18} \mathrm{O}$ value is somewhat higher than that of the next older and younger specimens from Teruel Basin (Fig. 5e), which could be due to higher temperatures (present-day mean annual, coldest month and warmest month temperatures in Valencia are 5.4, 6.6 and $3.8{ }^{\circ} \mathrm{C}$ higher than in Teruel) and the contribution of Mediterranean air masses.

A linear regression of all $\delta^{18} \mathrm{O}$ values against time results in a significant decrease of $0.30 \% / \mathrm{Myr}(p<0.0005, n=232, \mathrm{VM}$ excluded). A regression on the locality means may introduce biases, because these values may not correspond to true annual means, since the measured profiles do not exactly correspond to a year. Because there are too few data points per tooth to construct reliable sinusoidal fits, we preferred to take the midpoint between summer and winter extremes as a better and more representative measure of mean annual temperature compared to the average of all points. The differences between mean and midpoint values are very small, however: the average absolute difference is $0.07 \%$ ofor $\delta^{13} \mathrm{C}$ and $0.11 \%$ 。 for $\delta^{18} \mathrm{O}$ (Table 2). Also in terms of present-day local temperatures the difference between the annual midpoint and mean is small, e.g. $0.8{ }^{\circ} \mathrm{C}$ for the town of Teruel. Regressions of the $\delta^{18} \mathrm{O}$ midpoints on time show a significant decrease as well. E.g. the regression of $\delta^{18} \mathrm{O}_{\mathrm{H} 2 \mathrm{O}}$ midpoints on time yields a coefficient of $0.41 \% / \mathrm{Myr}$, which is significant at the $99 \%$ level $(p=0.009)$. In this calculation, we only used the eight ontogenetically sampled teeth from the Teruel and Calatayud-Daroca basins that show an approximately sinusoidal pattern (i.e. one tooth from Nombrevilla and the teeth from Masía del Barbo and Orrios are excluded, see also Section 4.4).

Certain localities or intervals show a considerable variation in isotopic composition. For instance, aberrant values occur for Los Mansuetos $(n=4)$ and Concud Cerro de la Garita $(n=3)$ (Fig. 5e). Both localities have an age of 7.2-7.1 Ma, suggesting a high level of climatic variability around that time. Three coexisting, differently-sized horse species in Las Casiones (6.3 Ma) show similar values suggesting that correlations of $\delta^{18} \mathrm{O}$ with phylogeny and body size are not very important. This observation corroborates earlier findings by Bryant et al. (1994).

Four factors may explain the decreasing trend in $\delta^{18} \mathrm{O}$ from $\sim 10 \mathrm{Ma}$ onwards: 1) a temperature decline due to regional topographic change, particularly paleolatitude and paleoaltitude, 2) a shift in the air mass source area resulting in more depleted precipitation, 3) a trend towards less evaporation, 4) long-term global cooling.

According to paleomagnetic reconstructions the paleolatitude of the study region was situated $\sim 2-4^{\circ}$ more south than today 10 million years ago, depending on the pole path chosen (Krijgsman and Tauxe, 2004; Torsvik et al., 2008; Langereis, pers. comm.). Using present-day $\delta^{18} \mathrm{O}$-latitude relationships for Iberia (e.g. Fig. 3 ) or the Northern Hemisphere in general (e.g. Fricke and O'Neil, 1999), this difference translates to a $1-1.4 \%$ onrichment, other things being equal. Paleolatitudinal change could therefore explain a significant portion $(\sim 25-40 \%)$ of the observed $\delta^{18} \mathrm{O}$ decrease and associated temperature decline, although it probably was less than this because of the stable land-sea distribution of the Iberian Peninsula.

No quantitative data exist on paleoaltitudes for the study region. Structural geological studies indicate that the general tectonic regime in the Iberian Chain region was dominated by compression until the 

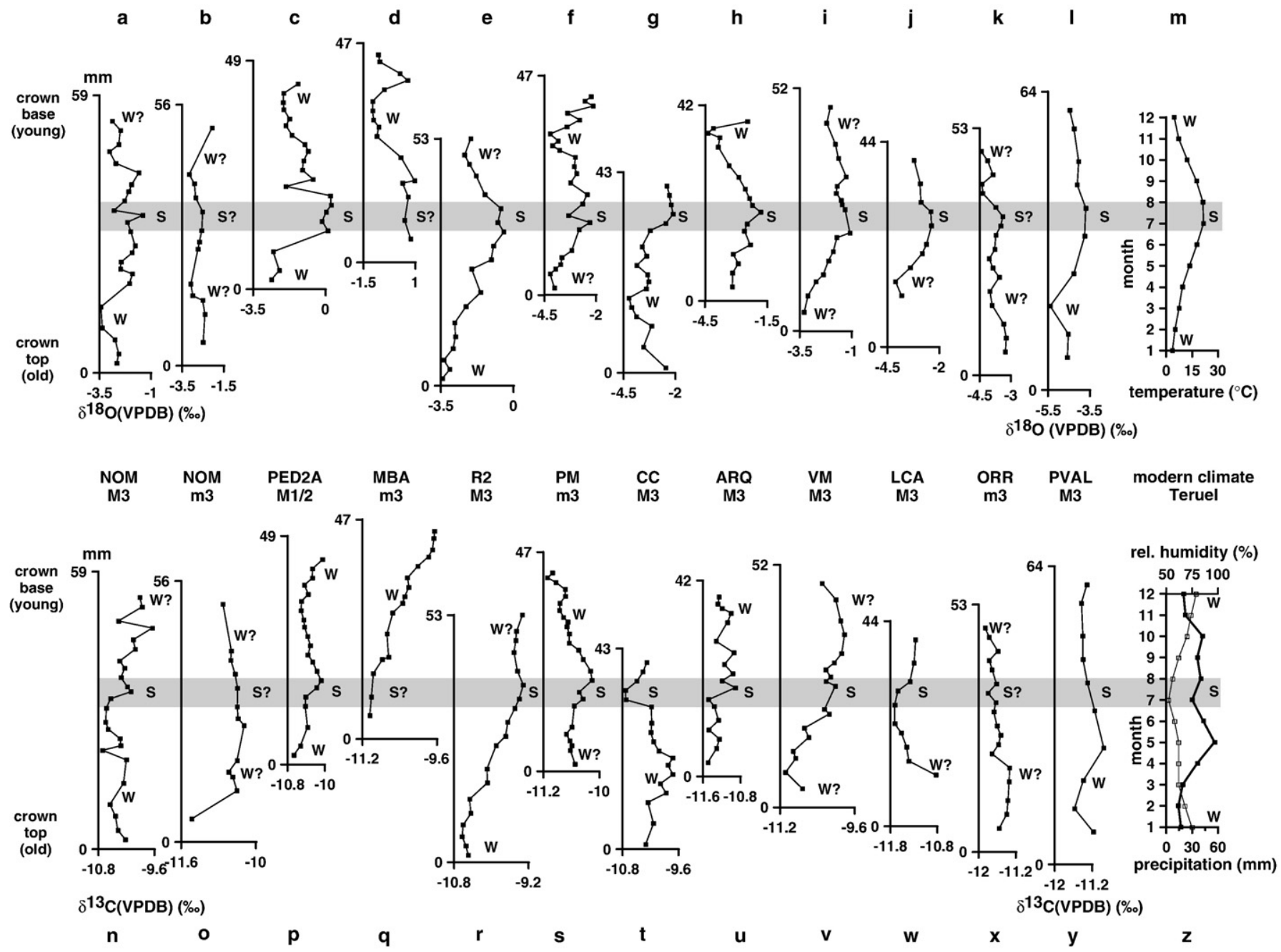

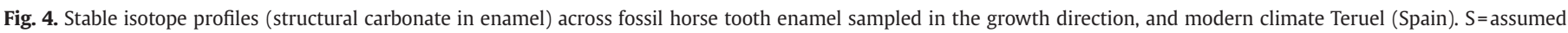

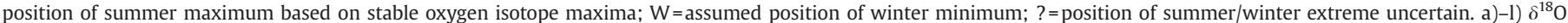

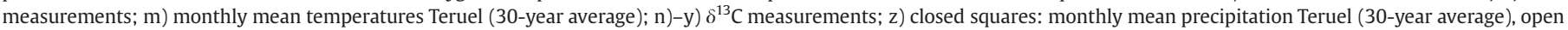
squares: relative humidity (30-year average).

Middle Miocene, and extension afterwards (Simón Gomez, 1983, 1991). During our period of study, the Late Miocene to Pliocene, tectonic activity would have been low (Casas-Sainz and Cortés-Gracia, 2002) and sedimentation would have kept up with rifting of the preCenozoic basement. This resulted in a late Pliocene $(\sim 3$ Ma old $)$ peneplain (Simón Gomez, 1983, 1991), which then would have been incised by the present-day river system. In addition, indications exist for a relatively young, Late Pliocene-Pleistocene phase of Iberian uplift, e.g. in the Central System (de Bruijne and Andriessen, 2002). Uplift could then have affected the relative isotopic results for the Late Pliocene locality of La Puebla de Valverde (2.1 Ma). For the rest of the interval (the Late Miocene to middle Pliocene, 11-3 Ma), we assume that changes in altitude were not big enough to have affected the oxygen isotope trend in any important way $(>1 \%$, i.e. $>\sim 300 \mathrm{~m}$ of altitude).

A decreasing trend of $\delta^{18} \mathrm{O}$ could also be explained by large-scale changes in the atmospheric circulation system itself. The detailed sedimentary record of the Mediterranean Sea has shown that the regional climate system, with precession being the dominant orbital cycle, has not changed in any fundamental way during the last 13.5 Myr (Hilgen et al., 2003). We therefore assume that air masses in Central Spain were mostly of Atlantic origin and controlled by the North Atlantic Oscillations (NAO), as they are today. The East Atlantic (EA) pattern, which results in the eastern advection of Mediterranean
Sea-derived moisture (Lionello et al., 2006), probably played a secondary role similarly as today. In a previous paper interpreting small-mammal community structure (van Dam, 2006) we inferred a drying step in Europe between 9-8 Ma and suggested that this step could have been controlled by a northward extension/shifting of the Subtropical High Pressure Zone, and a transition towards less zonal circulation. This process could ultimately have been triggered by uplift of the Tibetan plateau, as suggested by climate modeling results simulating the atmospheric effects of this uplift. Western source directions of air masses could have become less dominant over the Mediterranean Sea during summer, although this effect would be mainly confined to its eastern part. No major shift towards a continental source area for precipitation was predicted for the western Mediteranean and Iberian Peninsula (Ruddiman and Kutzbach, 1989).

The third possibility, a long-term reduction in evaporation, is not very likely. Firstly, the estimated mean $\delta^{18} \mathrm{O}_{\mathrm{H} 2 \mathrm{O}}$ values are mostly negative (mean:- $4.9 \%$; standard deviation: $1.7 \%$ ) and do not point to a distinct evaporative regime. Neither do the values measured on a series of different carbonate types (lacustrine, palustrine, paleosol, tufa) from different Late Miocene and Pliocene ages in the Teruel Basin, most of which have their $\delta^{18} \mathrm{O}$ in the range between -7 to $-5 \%$ (Alonso Zarza and Calvo, 2000). Secondly, the direction of change is opposite to precipitation predictions based on small mammal community 
GLOBAL

EAST CENTRAL SPAIN

stable isotopes tooth enamel
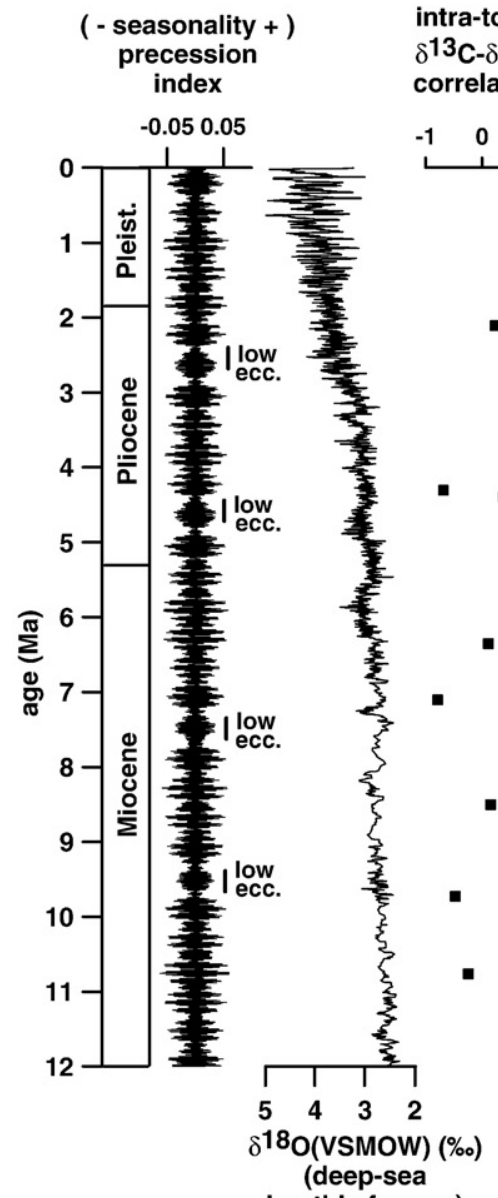

(deep-sea intra-tooth

correlation

$\begin{array}{lll}-1 & 0 & 1\end{array}$

C3 vegetation

average water-stressed
SPAIN

climatic interpretations
NW GERMANY - S NETHERLANDS

flora - based temperature mean annual precipitation $(\mathrm{mm})$ (small mammal - based)

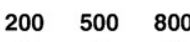

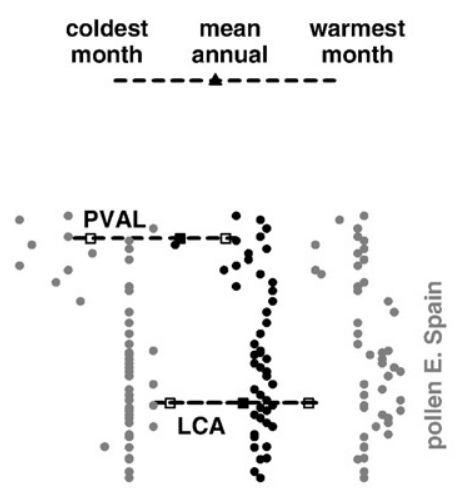

$\begin{gathered}\text { coldest mean } \\ \text { month annual } \\ \text { annest }\end{gathered}$
month

PVAL

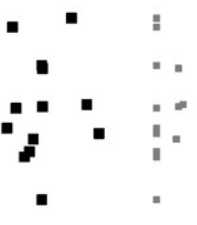

$\infty+$

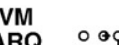

-

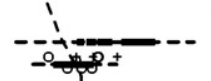

1

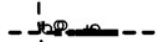

-

-
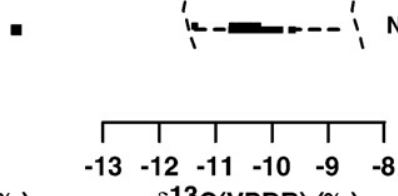

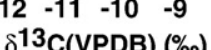

d

C

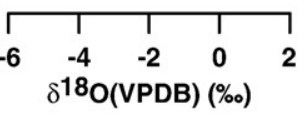

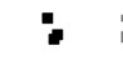

- r : :

0

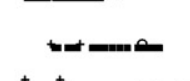

$++$

-

disappearance Dryopithecus Spain - ? PED2A

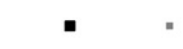

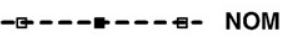

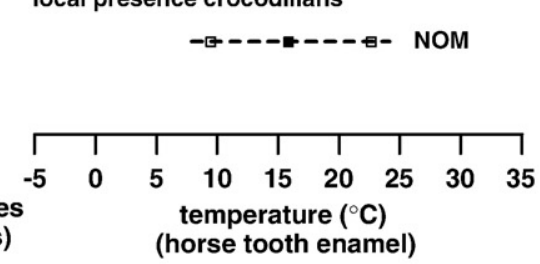

(horse tooth enamel)

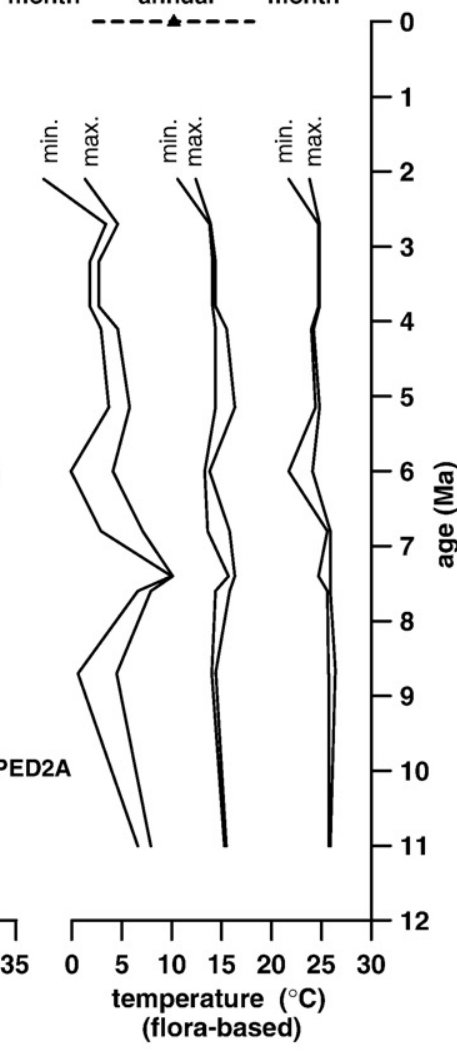

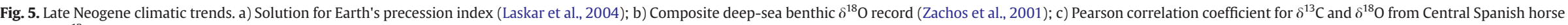

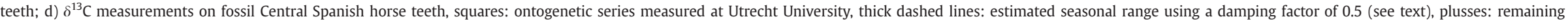

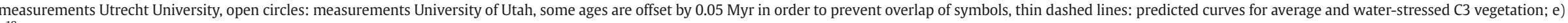

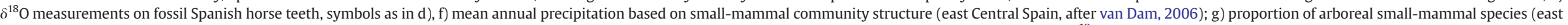

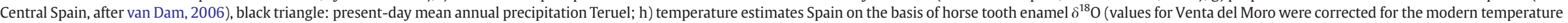

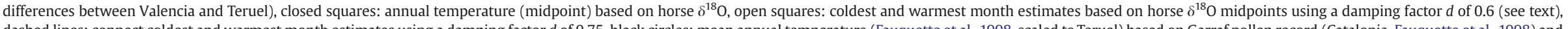

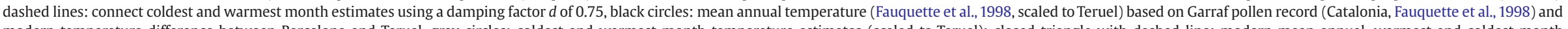

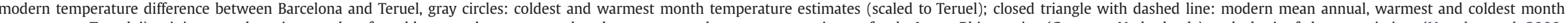

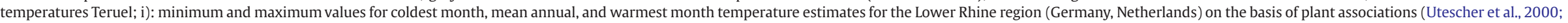

Mosbrugger et al., 2005), closed triangle with dashed line: modern mean annual, warmest and coldest month temperatures for Düsseldorf (NW Germany). 
structure, which indicate a drying step between 9-8 Ma (van Dam, 2006; see Fig. $5 \mathrm{f}$ and g).

In order to evaluate the fourth factor, global temperature change, we applied Eqs. (7) and (9) to the estimated $\delta^{18} \mathrm{O}_{\mathrm{H} 2 \mathrm{O}}$ values and compared the estimated temperatures to other proxy values (Fig. 5b,h and i). Note that our estimations include a small correction for ice-induced changes in seawater $\delta^{18} \mathrm{O}$ (data from Lear et al., 2000, see Table 2). The estimated temperature decrease appears to be statistically significant (LS regression) when based on the tooth-based midpoints of Fig. 5h. The average decrease is $0.84{ }^{\circ} \mathrm{C} / \mathrm{Myr}$ ( $p=0.010, n=8$, Venta del Moro excluded) or $0.83^{\circ} \mathrm{C} / \mathrm{Myr}(p=0.011, n=9$, geographically corrected value for Venta del Moro included). The mean estimated annual midpoint temperature based on the three 11-9 Ma old teeth (Vallesian Continental Stage) is $17{ }^{\circ} \mathrm{C}$ (Table 2), implying a $\sim{ }^{\circ} \mathrm{C}$ drop during the last 10 million years. (Note that $\sim 1-2{ }^{\circ} \mathrm{C}$ could be associated with northward plate tectonic migration, as described above). The tooth from the locality Masía del Barbo $2 \mathrm{~A}(9.7 \mathrm{Ma})$ shows a very high mean $\delta^{18} \mathrm{O}_{\mathrm{PO} 4}$ value of $21.6 \%$ 。 (Table 2), which would translate to an annual temperature of $23^{\circ} \mathrm{C}$. This value is probably an overestimation, because the reconstructed positive $\delta^{18} \mathrm{O}_{\mathrm{H} 2 \mathrm{O}}$ values (+1-2\%) for a number of sub-samples seem to point to evaporative enrichment during summer (Table 2). In addition, the isotopic profile of the MBA tooth (Fig. 4d) is not sinusoidal and difficult to interpret seasonally in the first place (see Section 4.4).

Temperature estimates for the Pliocene on the basis of the Garraf pollen core, collected a few kilometres southeast of Barcelona on the continental shelf (Fauquette et al., 1998), offer a possibility to compare estimated temperatures at $4-4.5 \mathrm{Ma}$, the age of the La Calera and Orrios teeth, and at $2 \mathrm{Ma}$, the age of La Puebla de Valverde. The pollen associations predict a rather stable early Pliocene value of $18{ }^{\circ} \mathrm{C}$ (Fauquette et al., 1998), i.e. $2.5{ }^{\circ} \mathrm{C}$ warmer than in Barcelona today. Assuming the same geographical temperature difference as today, MAT in Teruel would have been $14.3^{\circ} \mathrm{C}$. The predicted temperatures for $\mathrm{La}$ Calera is very similar: $14.6{ }^{\circ} \mathrm{C}$. The intra-tooth pattern for Orrios precludes the establishment of a reliable annual value (Fig. 4k). If the sub-sample mean would be taken as a representative value, the resulting temperature would be $12^{\circ} \mathrm{C}$, i.e. today's value. The estimate of $9{ }^{\circ} \mathrm{C}$ for La Puebla de Valverde (2.1 Ma) is lower than most time-equivalent pollenbased estimates of Fauquette et al. (1998), although a temperature down to $8^{\circ} \mathrm{C}$ (after spatial correction) has been predicted for one pollen sample of approximately this age (Fig. $5 \mathrm{~h}$ ). Flora-based MAT estimates from the Lower Rhine Basin (Germany, Netherlands) for the interval 4-3 Ma (Hambach) and $2 \mathrm{Ma}$ (Tegelen) are $14-15$ and $11-12{ }^{\circ} \mathrm{C}$, respectively, or $4-5^{\circ} \mathrm{C}$ and $1-2{ }^{\circ} \mathrm{C}$ warmer than today (Utescher et al., 2000; Mosbrugger et al., 2005). Because the mammal-based temperature predictions fit other estimates fairly well, we hypothesize that the observed decreasing trend in horse tooth $\delta^{18} \mathrm{O}$ in east Central Iberia largely reflects the global late Cenozoic cooling trend. Obviously, more data are needed to substantiate this hypothesis.

\section{3. $\delta^{13} \mathrm{C}$ results}

$\delta^{13} \mathrm{C}$ values show a decreasing trend as well, from values around -10 to $-10.5 \%$ in the early Late Miocene to values around $-11.5 \%$ in the Pliocene (Fig. 5d). A linear regression of all $\delta^{13} \mathrm{C}$ values against time shows a significant decrease of $0.15 \% / \operatorname{Myr}(p<0.0005$, Venta del Moro excluded). A regression using the means for the 11 ontogenetically sampled teeth yields a significant decrease as well $(p=0.005)$. All $\delta^{13} \mathrm{C}$ values are in the range corresponding to a diet of C3 plants. Theoretically, variation in $\delta^{13} \mathrm{C}$ of $\mathrm{C} 3$ vegetation may be as large as $13 \%$ o depending on factors such as light level, position in the canopy and water stress (Farquhar et al., 1989; Van der Merwe and Medina, 1991; Ehleringer and Monson, 1993; see also Koch, 1998; Kohn and Cerling, 2002). Passey et al. (2002) have used estimations of Neogene trends in $\delta^{13} \mathrm{C}_{\mathrm{CO} 2}$ on the basis of planktonic foraminifers (e.g. Pagani et al., 1999) to predict $\delta^{13} \mathrm{C}$ "null" curves for average and water-stressed vegetation (Fig. $5 \mathrm{~d}$ ). The horse enamel $\delta^{13} \mathrm{C}$ sequence appears to follow the predicted curves, including a slight drop at the MioPliocene transition, and points to a diet which is closer to an average C3 diet than to a "water-stressed" C3 diet.

\subsection{Intra-tooth variability and seasonality}

Most teeth show quasi-sinusoidal isotopic variations in their $\delta^{18} \mathrm{O}$ (Fig. 4a-1), which we attribute to seasonality in temperature, with the lowest values occurring in winter (see Fig. 3). The approximate positions of maxima and maxima are indicated by $\mathrm{S}$ (summer) and W (winter) in Fig. 4, respectively. Question marks (S?, W?) signify that summer and winter extremes cannot be recognized with certainty, either because the sinusoidal pattern is not clear, or because the extremes are close to the crown base or top. A short reversal near the crown base occurs in four specimens (Fig. 4c, e, h and i). The first three of these are worn teeth, with the reversals most probably corresponding to the reduction in enamel formation rate that occurs after eruption. Three out of the four teeth that do not show a clear sinusoidal pattern are lower third molars (from Nombrevilla, Masía del Barbo and Orrios). There is no reason to believe that lower teeth responded differently than upper teeth, since the development of corresponding tooth pairs is known to run reasonably synchronously (Hoppe et al., 2004a,b, and references therein). We note that Nelson (2005) also found a number of aberrant patterns in Hipparion that did not conform to the expected sinusoidal shape.

In six teeth the position of both a summer and winter extreme can be established with relatively high precision (Fig. 4a, c, f, g, h and 1). From these teeth yearly growth rates can be estimated, assuming that the time between these extremes corresponds to half a year. These rates are 46 mm/yr (M1/2, Pedregueras 2C), 44 mm/yr (M3, Nombrevilla) and $32 \mathrm{~mm} / \mathrm{yr}$ (m3, Puente Minero) for Hipparion primigenium, $40 \mathrm{~mm} / \mathrm{yr}$ (M3, Concud) for H. concudense), $36 \mathrm{~mm} / \mathrm{yr}$ (M2, El Arquillo) for $H$. sp., and $40 \mathrm{~mm} / \mathrm{yr}$ (M3, La Puebla de Valverde) for Equus stenonis. Similar rates were found in other horse tooth isotope studies (Sharp and Cerling, 1998; Fricke et al., 1998; Kohn et al., 1998; Passey et al., 2002; Kohn and Cerling, 2002; Hoppe et al., 2004b; Nelson, 2005).

The total duration of mineralization of (lower) third molars in modern Equus has been estimated to be 34 months, starting 1824 months after birth (Hoppe et al., 2004b). Given the above mentioned growth rates for Hipparion third molars, mineralization in these teeth would certainly not have exceeded two years. Most $\delta^{18} \mathrm{O}$ profiles in Fig. 4 show winter values at the crown base, i.e. where the youngest enamel has formed. Assuming that the young Hipparion horses were born in spring, as is the case for most Equus caballus foals today (Nowak, 1999), mineralization of the third molars would have stopped between 1.5 and 2 years after birth, i.e. earlier than in Equus caballus today. Using overlapping isotopic ranges of different dental elements, also Nelson (2005: Fig. 7) estimated the period between birth and end of mineralization of the third molars (and hence in the dentition) to be $\sim 2$ years in Hipparion, with birth probably taking place in the wet season.

The estimated intra-tooth $\delta^{18} \mathrm{O}_{\mathrm{PO} 4}$ variation in the 12 ontogenetically sampled teeth from Central Spain varies between 1.1 and 3.0\%。 (VSMOW, Table 2$)$. The largest amplitudes ( 3.0\%) are observed in teeth of early Late Miocene age (10.0 and 9.2 Ma). Seasonal ranges in $\delta^{13} \mathrm{C}$ vary between 0.5 and $1.4 \%$ ( (Table 2 ). Again, the largest amplitudes $(>1.3 \%$ ) occur in early Late Miocene teeth (9.7 and 9.2 Ma). Half of the teeth show positive $\delta^{18} \mathrm{O}-\delta^{13} \mathrm{C}$ correlations (between 0.11 and 0.80 ) and half show negative correlations $(-0.25$ to -0.79$)$ (Fig. $5 \mathrm{c}$ ).

In order to estimate seasonal $\delta^{18} \mathrm{O}_{\mathrm{H} 2 \mathrm{O}}$ and temperature ranges, the observed ranges have to be divided by $(1-d)$, with $d$ being the damping factor. As shown above, the prediction of these ranges is hampered by the uncertainties in $d$, as outlined above. The theoretical simulations above (Fig. 2) point to a effective damping factor of 0.60.75 depending on sample resolution. In principle it would be possible 
to constrain the magnitude of $d$ by evaluating the predictions of the within-year $\delta^{18} \mathrm{O}_{\mathrm{H} 2 \mathrm{O}}$ and temperature ranges and extremes against predictions based on other sorts of data. As both climate and isotopic data are usually given as monthly means and our sub-samples corresponds to $\sim 1$ week in the direction of growth, the appropriate $d$ value to convert the isotopic back to environmental amplitude would be in the order of $\sim 0.6$, based on the data in Fig. 2. In Table 2, we show the consequences for $\delta^{18} \mathrm{O}_{\mathrm{H} 2 \mathrm{O}}$ and temperature ranges for a $d$ value of 0.6 and a for more extreme value of 0.75 .

\section{Discussion}

\subsection{Late Neogene cooling}

Assuming that the observed drop in horse tooth enamel $\delta^{18} \mathrm{O}$ reflects Cenozoic cooling, temperatures in Central Spain would have dropped $\sim 5^{\circ} \mathrm{C}$ between $11 \mathrm{Ma}$ and today (Fig. $5 \mathrm{~h}$ ). This decrease is similar to the mean annual temperature drop of $\sim 6{ }^{\circ} \mathrm{C}$ estimated for the Lower Rhine basin (Germany, Netherlands) on the basis of plant associations (e.g. Utescher et al., 2000; Mosbrugger et al., 2005). Deep-ocean temperatures are supposed to have cooled somewhat less since $11 \mathrm{Ma}\left(3-4{ }^{\circ} \mathrm{C}\right.$, Lear et al., 2000; deep-sea $\delta^{18} \mathrm{O}$ record of Zachos et al. (2001) shown in Fig. 5b). North American horses show a similar 2-3\% drop in $\delta^{18} \mathrm{O}$ values between $11 \mathrm{Ma}$ and modern times. This decrease has been attributed to climatic change as well (Passey et al., 2002) although in some regions tectonics probably played a more important role via the altitude effect (Kohn et al., 2002). Remarkably, crocodilians disappear about the same time ( $10 \mathrm{Ma}$ ) from both Nebraska, US (Passey et al., 2002) and Central Teruel, Spain. The finding of a 9.9 Ma old crocodilian tooth in the Teruel Basin (Masía de la Roma 4B, Boehme et al., 2006) is consistent with high mean annual temperatures $\left(20^{\circ} \mathrm{C}\right.$ or higher, Table 2, Fig. $\left.4 \mathrm{~h}\right)$ around this time. The presence of a warm climate in Spain at this time is also corroborated by the last European incidence of representatives of the fruit-eating great ape Dryopithecus (Can Llobateres, Vallès-Penedès Basin, 9.8 Ma, Fig. 4h), which would have required an environment characterized by a certain proportion of ever-green tropical plants (Agusti et al., 2003). The inference of high temperatures between 11$9 \mathrm{Ma}$ in the Teruel basin is not in agreement with reconstructions of relative temperatures on the basis of relative abundances and latitudinal distribution of rodent groups, which show lower values than during the 9-6 Ma interval (van Dam and Weltje, 1999). The reason could be that most (small-)mammal distribution areas in low to middle latitudes are controlled by precipitation rather than by temperature (van Dam, 2006). Estimations of rainfall based on the proportions of wet-adapted (arboreal, insectivorous) small mammals indicate an overall drying trend, the occurrence of which is consistent with climatic cooling (van Dam, 2006; Fig. $5 f$ and g).

When Eq. (4) is applied to Late Pliocene and Pleistocene horse tooth enamel $\delta^{18} \mathrm{O}_{\mathrm{PO} 4}$ data from Southern Spain (Guadix-Baza and Granada Basins; altitude $900 \mathrm{~m}$; seven localities with 6-9 specimens, Sanchez Chillón, 1993; Sánchez-Chillón et al., 1994), $\delta^{18} \mathrm{O}_{\mathrm{H} 20}$ values of $-8.5 \%$ result at $2.7 \mathrm{Ma},-7 \%$ at $1.3 \mathrm{Ma},-10$ to $-9 \%$ at $1.3-0.8 \mathrm{Ma}$, and $-8 \%$ o at $0.2 \mathrm{Ma}$. These estimates are much lower than the mean composition of rainfall measured in the nearby stations of Gibraltar (-4\%, Fig. 3a) and Sevilla $(\sim-3 \%)$ today. Converting the fossil $\delta^{18} \mathrm{O}$ compositions to temperature yields values in the range of $5-8{ }^{\circ} \mathrm{C}$ for most localities, i.e. $\sim 10^{\circ} \mathrm{C}$ colder than today. Such temperatures seem too low, favoring the explanation that the isotopic compositions are depleted by excess light oxygen added via the melting of isotopically light snow in the nearby Sierra Nevada mountains (Sánchez-Chillón et al., 1994).

\subsection{Vegetation}

The measured $\delta^{13} \mathrm{C}$ values around -11 and $-10 \%$ point to the presence of an average or slightly water-stressed C3 type of vegetation (Passey and Cerling, 2002). We therefore conclude that C4 vegetation was virtually absent in east Central Spain during the late Miocene and Pliocene. Also in Eastern Spain C3 vegetation was probably the dominant type, as evidenced by a $-12.0 \%$ ovalue measured on Spanish Hipparion from Seo de Urgell, Vallès-Penedès basin, E. Spain, 11$10 \mathrm{Ma}$, Cerling et al. (1997), Cerling, pers. comm.). The absence of C4 vegetation in the Eastern Mediterranean region in this period was already indicated by studies of mammal tooth and paleosol carbonate $\delta^{13} \mathrm{C}$ in Greece and Turkey (Bocherens et al., 1994; Quade et al., 1994; Bocherens and Sen, 1998). The lack of C4 grasses in the Mediterranean region can be explained by the presence of a Mediterranean-type of climate with a dry summer, lacking a growing season which is wet and warm at the same time. Despite the absence of $C 4$ grasses, the mammal community structure in the Teruel Basin points to an open landscape (woodland) during most of the studied interval (Alcalá, 1994). Long periods of absence of arboreal small mammals (especially from 9 Ma onwards, Fig. $5 \mathrm{~g}$ ) also point to open vegetation.

As mentioned above, our $\delta^{13} \mathrm{C}$ tooth record shows a decreasing trend that seems to track global $\delta^{13} \mathrm{C}_{\mathrm{CO} 2}$ (Passey and Cerling, 2002) implying a relatively stable local vegetation (structure). A comparable trend in tooth $\delta^{13} \mathrm{C}$ can be seen in the European (mainly French) data published by Cerling et al. (1997: their Fig. 2).

\subsection{Seasonality}

Most Late Miocene intra-tooth $\delta^{18} \mathrm{O}$ and $\delta^{13} \mathrm{C}$ ranges are larger (up to twice a large) than the Pliocene ranges, (Table 2, Fig. $5 \mathrm{~d}$ and e). A similar Mio-Pliocene decrease in amplitude has been noted on the basis of North American horse teeth (Kohn et al., 2002). Whether this parallel trend points to a wide-spread, reduced Early Pliocene seasonality cannot be stated with certainty, but it would be consistent with the existence of the so-called Pliocene climatic optimum (e.g. Raymo et al., 1996). The Pakistan Late Miocene intra-tooth horse record of Nelson (2005) shows a constant amplitude of $2-3 \%$ in $\delta^{18} \mathrm{O}$, which is comparable to that in the Spanish horses (mean of 2.1\%o). This similarity in $\delta^{18} \mathrm{O}$ variability does not imply similarity in climate, however, because the late Miocene climate in Pakistan is thought to have been monsoonal, with precipitation amount instead of temperature controlling intra-tooth oxygen isotopic variability (amount effect, Dettman et al., 2001; Nelson, 2005). The mean estimated annual $\delta^{18} \mathrm{O}_{\mathrm{H} 2 \mathrm{O}}$ range on the basis of our observed tooth profiles is $5.6 \%$ o using $d=0.6$, and $6.9 \%$ o using $d=0.75$ (Table 2). Both values are close to the present-day ranges of 7.3 and $5.4 \%$ for the nearby modern reference stations Zaragoza and Valencia, respectively. The corresponding mean estimated temperature ranges are 15 and $18{ }^{\circ} \mathrm{C}$. The latter estimate is the same as the modern annual range for Teruel today. A weak constraint on $d$ might be inferred from the occurrence of strongly enriched, positive summer $\delta^{18} \mathrm{O}_{\mathrm{H} 2 \mathrm{O}}$ maxima of $+1-2 \%$ (i.e. in the evaporative regime) for $d=0.75$ for the early Late Miocene locality Pedregueras 2A, and of even higher values in Masía del Barbo 2A. Such values appear to be too high in comparison to values of $-6 \%$ measured in various types of local carbonate rocks from this interval (Alonso Zarza and Calvo, 2000) and to precipitation reconstructions based on small-mammal community structure (van Dam, 2006; Fig. 5f).

Fauquette et al. (1998) estimated Pliocene coldest and warmest month values for the off-shore Garraf pollen core. Their seasonal ranges are 20 and $22^{\circ} \mathrm{C}$ before and after $2.8 \mathrm{Ma}$, respectively (Fig. 4h), i.e. much larger than today $\left(15^{\circ} \mathrm{C}\right.$ in Barcelona). As noted above, the Pliocene ranges estimated from the teeth are relatively low: $11^{\circ} \mathrm{C}$ using $d=0.6$ or $14^{\circ} \mathrm{C}$ using $d=0.75$ for both localities La Calera and La Puebla de Valverde. The difference with the pollen-based estimates is caused by a lower summer temperature (Fig. 5h). Part of the discrepancy could be explained by the use of midpoint instead of mean values, and/or by the fact that mid-latitude correlations of $\delta^{18} \mathrm{O}_{\mathrm{H} 2 \mathrm{O}}$ with summer temperature are weaker than with winter temperature (Fricke and O'Neil, 1999). Evidently, more precise measurements and models of seasonality in tooth enamel $\delta^{18} \mathrm{O}$ as well as reconstructions based on 
other sorts of data are needed to refine the estimates of within-year $\delta^{18} \mathrm{O}_{\mathrm{H} 2 \mathrm{O}}$ and temperature amplitudes.

The measured intra-tooth $\delta^{13} \mathrm{C}$ amplitudes should be corrected for damping as well. In Table 2 and Fig. $5 d$ we use $d=0.5$ (i.e. amplification by 2.0) because there is no direct mixing with the atmosphere, as is the case for oxygen. The resulting mean summer-winter difference is $1.9 \%$, with amplitudes generally becoming smaller through time. Seasonal variations in $\delta^{13} \mathrm{C}_{\mathrm{CO} 2}$ explain part of this variation: because of the presence of a larger $\left({ }^{12} \mathrm{C}\right.$-favoring) Northern Hemisphere plant mass, Northern Hemisphere summer values are relatively enriched in ${ }^{13} \mathrm{C}$. The modern intra-annual variation is in the order of $1.0 \%$ (Francey et al., 1995). The remaining part of the seasonal $\delta^{13} \mathrm{C}$ amplitude should be attributed to a combination of seasonality in microenvironmental factors such as water-stress and light level that affect photosynthesis in the $\mathrm{C} 3$ vegetation, to the mode and timing of growth of the consumed plant species, and/or to seasonal changes in the dietary preferences of the horses. Half of the teeth show higher $\delta^{13} \mathrm{C}$ values during summer (as inferred on the basis of $\delta^{18} \mathrm{O}$, Fig. 4). Summer enrichment would be expected because of the above-mentioned higher global $\delta^{13} \mathrm{C}_{\mathrm{CO} 2}$ values during Northern Hemisphere summers, and because of a higher degree of water stress (e.g. Ehleringer et al., 1992; Barbour et al., 1999; Walcroft et al., 2002). Although modern summer precipitation in Teruel is higher than winter precipitation, relative humidity is not (Fig. $4 z$ ), because of the high summer temperatures.

Intervals displaying a negative intra-tooth correlation between $\delta^{18} \mathrm{O}$ and $\delta^{13} \mathrm{C}$ (Fig. 5c) could have been characterized by less warm summers, and/or warmer winters. Such conditions of reduced seasonality would for example apply to astronomical configurations characterized by a reduced precession effect of the Earth's axis during low eccentricity, i.e. when the Earth moves around the Sun in an almost circular orbit (Fig. 4a). Such eccentricity minima have been recognized in the basin lithology and appear to correlate to faunal change (van Dam et al., 2006). Some localities showing negative isotopic O-C correlations (Masía del Barbo 2A, Orrios) do correlate to long-term eccentricity minima (Fig. $3 \mathrm{a}$ and $\mathrm{b}$ ). Because the beds with the mammal sites are generally thought to correlate to precession minima (Abdul Aziz et al., 2004; Abels, personal communication) loweccentricity sites would have been characterized by cooler summers compared to high-eccentricity sites. Clearly, additional sampling of levels and specimens is necessary to check the relation between climatic regime and seasonal isotopic signature.

\section{Summary and conclusions}

Stable oxygen and carbon isotopic data from fossil horse tooth enamel provide valuable paleoenvironmental information, especially when complete tooth profiles are analyzed. Because horses have high drinking rates, their tooth $\delta^{18} \mathrm{O}$ can be used to reconstruct $\delta^{18} \mathrm{O}$ values of their drinking water and the local precipitation. These, in turn, can be used to predict temperatures at mid to high latitudes, because of the cumulative preferential outraining of ${ }^{18} \mathrm{O}$ due to cooling in travelling air masses.

Our compilation of the oxygen isotope compositions from the structural carbonate of the tooth enamel of late Neogene (11-2 Ma) horse teeth (mainly Hipparion) from east Central Spain shows a decreasing trend in $\delta^{18} \mathrm{O}$ between $10 \mathrm{Ma}$ and today. This drop is equivalent to a $5{ }^{\circ} \mathrm{C}$ mean annual temperature decrease, using modern relationships between horse $\delta^{18} \mathrm{O}$ and local water $\delta^{18} \mathrm{O}$, and between Iberian local water $\delta^{18} \mathrm{O}$ and temperature data. The estimated temperature change is similar to that based on Central European floras. The occurrence of a significantly warmer climate 10 million years ago is corroborated by the local occurrence of crocodilians and the regional presence of hominids and tropical plant species. Although changes in paleolatitude, paleolaltitude, evaporation, and air mass source area will have affected $\delta^{18} \mathrm{O}$ as well, these effects were probably secondary to that of late Cenozoic cooling.
The observed within-tooth $\delta^{18} \mathrm{O}$ variability almost certainly reflects seasonality in temperature. Although the estimated seasonal temperature ranges are in the expected range, the current uncertainties with regard to the amount of damping due to buffering and time averaging preclude very accurate reconstructions of these ranges. The minimum amount of damping due to the combined internal effects of mixing with atmospheric $\mathrm{O}_{2}$, reservoir dynamics, enamel maturation and sampling can be estimated to be $\sim 60 \%$ at the monthly level.

The stable carbon isotopic record indicates the virtual absence of C4 plants in east Central Spain, thereby confirming earlier ideas that C4 plants did not invade Europe and the Mediterranean region during the Late Neogene. The long-term, slightly decreasing trend in $\delta^{13} \mathrm{C}$ follows the expected drop in average global $\delta^{13} \mathrm{C}_{\mathrm{CO} 2}$ and therefore may not reflect changes in the local environment. Within-tooth $\delta^{13} \mathrm{C}$ variation is small and can be attributed to a combination of seasonal changes in global $\delta^{13} \mathrm{C}_{\mathrm{CO} 2}$ and in local factors related to climate (e.g. aridity), vegetation composition / structure and dietary preferences. Orbitallyforced variations in seasonality will undoubtedly have affected the isotopic values and may partly explain the occurrence of either positive or negative intra-tooth $\delta^{18} \mathrm{O}-\delta^{13} \mathrm{C}$ correlations.

\section{Acknowledgements}

The research was funded by the Department of Earth Sciences, Utrecht University, the Netherlands. We thank A. van Dijk for laboratory assistance, L. Alcalá from the Fundación Conjunto Paleontológico de Teruel-Dinópolis, Spain, and B. Sanchez-Chillón from the Museo Nacional de Ciencias Naturalis Madrid, Spain for making available fossils for isotopic analysis. We are grateful to T. Cerling from the University of Utah for supplying us with unpublished horse tooth stable isotope data, and to B. Sanchez-Chillón for sampling these teeth. We thank C. Langereis and W. Krijgsman for providing us with paleolatitude estimates. We thank T. Tütken from the University of Bonn and two anonymous reviewers for their helpful comments.

\section{References}

Abdul Aziz, H., van Dam, J.A., Hilgen, F., Krijgsman, W., 2004. Astronomical forcing in Upper Miocene continental sequences: implications for the geomagnetic polarity time scale. Earth Planet. Sci. Lett. 222, 243-258.

Agusti, J., Sanz de Siria, A., Garcés, M., 2003. Explaning the end of the hominoid experiment in Europe. J. Hum. Evol. 45, 145-153.

Alberdi, M.T., 1974. El género Hipparion en España. Nuevas formas de Castilla y Andalucía, revisión e historia evolutiva. Trab. Neogeno-Cuatern. 1, 1-146.

Alberdi, M.T., Alcalá, L., 1990. El género Hipparion en la fosa de Alfambra Teruel. Paleoantol. Evol. 23, 105-109.

Alcalá, L., 1994. Macromamíferos neógenos de la fosa de Alfambra-Teruel. Instituto de Estudios Turolenses-Museo Nacional de Ciencias Naturales, Teruel.

Alonso Zarza, A.M., Calvo, J.P., 2000. Palustrine sedimentation in an episodically subsiding basin: the Miocene of the northern Teruel Graben. Palaeogeogr. Palaeoclimatol. Palaeoecol. 160, 1-21.

Barbour, M.M., Walcroft, A.S., Farquhar, G.D., 1999. Seasonal variation in $\delta^{13} \mathrm{C}$ and $\delta^{18} \mathrm{O}$ of cellulose from growth rings of Pinus radiata. Plant Cell Environ. 20,1543-1550.

Besems, R.E., Van de Weerd, A., 1983. The Neogene rodent biostratigraphy of the TeruelAdemuz Basin (Spain). Kon. Ned. Akad. Wetensch. Proc. B 86, 17-24.

Bocherens, H., Sen, S., 1998. Isotopic investigation In: Sen, S. (Ed.), Pliocene vertebrate locality of Çalta, Ankara, Turkey. Geodiversitas, vol. 20, pp. 487-495.

Bocherens, H., Fizet, M., Mariotti, A., Bellon, G., Borel, J.-P., 1994. Les gisements de mammifères du Miocène supérieur de Kemiklitepe, Turquie: 10. Biogéochimie isotopique. Bull. Mus. Natl. Hist. Nat., Paris 16 C, 211-223 4e sér.

Boehme, M., Ilg, A., Ossig, A., Küchenhoff, H., 2006. New method to estimate paleoprecipitation using fossil amphibians and reptiles and the middle and late Miocene precipitation gradients in Europe. Geology 34, 425-428.

Bryant, J., Luz, B., Froelich, P., 1994. Oxygen isotopic composition of fossil horse tooth phosphate as a record of continental paleoclimate. Palaeogeogr. Palaeoclimatol. Palaeoecol. 107, 303-316.

Bryant, J., Froelich, P., Showers, W., Genna, B., 1996a. Biological and climatic signals in the oxygen isotopic composition of Eocene-Oligocene equid enamel phosphate. Palaeogeogr. Palaeoclimatol. Palaeoecol. 26, 75-89.

Bryant, J., Froelich, P., Showers, W., Genna, B., 1996b. A tale of two quarries: biologic and taphonomic signatures in the oxygen isotopic composition of tooth enamel phosphate from modern and Miocene equids. Palaios 11, 397-408.

Casas-Sainz, A.M., Cortés-Gracia, A.L., 2002. Cenozoic landscape development within the Central Iberian Chain, Spain. Geomorphology 44, 19-46. 
Celle-Jeanton, H., Travi, Y., Blavoux, B., 2001. Isotopic typology of the precipitation in the Western Mediterranean region at three different time scales. Geophys. Res. Lett. 28, 1215-1218.

Cerling, T.E., Harris, J.M., MacFadden, B.J., Leakey, M.G., Quade, J., Eisenmann, V., Ehleringer, J.R., 1997. Global vegetation change through the Miocene/Pliocene boundary. Nature 389, 153-158.

Cole, J.E., Rind, D., Webb, R.S., Jouzel, J., Healy, R., 1999. Climatic controls on interannual variability of precipitation $\delta^{18} \mathrm{O}$ : Simulated influence of temperature, precipitation amount, and vapor source region. J. Geophys. Res.-Atmos. 104, 14223-14235.

Dansgaard, W., 1964. Stable isotopes in precipitation. Tellus 16, 436-468.

de Bruijne, C.H., Andriessen, P.A.M., 2002. Far field effects of Alpine plate tectonism in the Iberian microplate recorded by fault-related denudation in the Spanish Central System. Tectonophysics 349, 161-184.

Delgado Huertas, A., Iacumin, P., Stenni, B., Sanchez Chillon, B., Longinelli, A., 1995. Oxygen isotope variations of phosphate in mammalian bone and tooth enamel. Geochim. Cosmochim. Acta 59, 4299-4305.

Dettman, D.L., Kohn, M.J., Quade, J., Ryerson, F.J., Ojha, T.P., Hamidullah, S., 2001. Seasonal stable isotope evidence for a strong Asian monsoon throughout the past 10.7 m.y. Geology 29, 31-34.

Domingo, L., Cuevas-González, J., Grimes, S.T., Hernández Fernández, M., LópezMartínez, N., 2009. Multiproxy reconstruction of the palaeoclimate and palaeoenvironment of the Middle Miocene Somosaguas site (Madrid, Spain) using herbivore dental enamel. Palaeogeogr., Palaeoclimatol., Palaeoecol. 272, 53-88.

Ehleringer, J.R., Monson, R.K., 1993. Evolutionary and ecological aspects of photosynthetic pathway variation. Ann. Rev. Ecolog. Syst. 24, 411-439.

Ehleringer, J.R., Phillips, S.L., Comstock, J.P., 1992. Seasonal variation in the carbon isotopic composition of desert plants. Funct. Ecol. 6, 396-404.

Farquhar, G.D., Ehleringer, J.R., Hubick, K.T., 1989. Carbon isotope discrimination and photosynthesis. Ann. Rev. Plant Physiol. Mol. Biol. 40, 530-537.

Fauquette, S., Guiot, J., Suc, J.-P., 1998. A method for climatic reconstruction of the Mediterranean Pliocene using pollen data. Palaeogeogr. Palaeoclimatol. Palaeoecol. 144, 183-201.

Feranec, R.S., MacFadden, B.J., 2000. Evolution of the grazing niche in Pleistocene mammals from Florida: evidence from stable isotopes. Palaeogeogr. Palaeoclimatol. Palaeoecol. 162, 155-169.

Francey, R.J., Tans, P.P., Allison, C.E., Enting, I.G., White, J.W.C., Trolier, M., 1995. Changes in oceanic and terrestrial carbon uptake since 1982. Nature 373, 326-330.

Fricke, H.C., O'Neil, J.R., 1999. The correlation between ${ }^{18} \mathrm{O} /{ }^{16} \mathrm{O}$ ratios of meteoric water and surface temperature: its use in investigating terrestrial climate change over geologic time. Earth Planet. Sci. Lett. 170, 181-196.

Fricke, H.C., Clyde, W.C., O'Neil, J.R., 1998. Intra-tooth variations in $\delta^{18} \mathrm{O}$ (PO4) of mammalian tooth enamel as a record of seasonal variations in continental climate variables. Geochim. Cosmochim. Acta 62, 1839-1850.

Friedman, I., O'Neil, J.R., 1977. Compilation of stable isotope fractionation factors of geochemical interest. U.S. Geological Survey, Reston.

Gadbury, C., Todd, L., Jahren, A., Amundson, R., 2000. Spatial and temporal variations in the isotopic composition of bison tooth enamel from the Early Holocene Hudson-Meng Bone Bed, Nebraska. Palaeogeogr. Palaeoclimatol. Palaeoecol. 157, 79-93.

Grimes, S.T., Mattey, D.P., Hooker, J.J., Collinson, M.E., 2003. Paleogene paleoclimate reconstruction using oxygen isotopes from land and freshwater organisms: the use of multiple paleoproxies. Geochim. Cosmochim. Acta 67, 4033-4047.

Hilgen, F.J., Abdul Aziz, H., Krijgsman, W., Raffi, I., Turco, E., 2003. Integrated stratigraphy and astronomical tuning of the Serravallian and lower Tortonian at Monte dei Corvi (Middle-Upper Miocene, northern Italy). Palaeogeogr. Palaeoclimatol. Palaeoecol. 199, 229-264.

Hoppe, K.A., 2006. Correlation between the oxygen isotope ratio of North American bison teeth and local waters: implication for paleoclimatic reconstructions. Earth Planet. Sci. Lett. 244, 408-417.

Hoppe, K.A., Amundson, R., Vavra, M., McClaran, M.P., Anderson, D.L., 2004a. Isotope analyses of equid teeth from modern North American feral horses: implications for paleoenvironmental reconstructions. Palaeogeogr. Palaeoclimatol. Palaeoecol. 203, 299-311.

Hoppe, K.A., Stover, S.M., Pascoe, J.R., Amundson, R., 2004b. Patterns of tooth enamel biomineralization in modern domestic horses: implications for isotopic microsampling. Palaeogeogr. Palaeoclimatol. Palaeoecol. 206, 355-365.

Iacumin, P., Bocherens, H., Mariotti, A., Longinelli, A., 1996. Oxygen isotope analyses of co-existing carbonate and phosphate in biogenic apatite; a way to monitor diagenetic alteration of bone phosphate? Earth Planet. Sci. Lett. 142, 1-6.

IEAE, 2005. 2002 GNIP data. http://isohis.iaea.org. 2005.

Jouzel, J., Russell, G.L., Suozzo, R.J., Koster, R.D., White, J.W.C., Broecker, W.S., 1987. Simulations of the HDO and $\mathrm{H}_{2}^{18} \mathrm{O}$ atmospheric cycles using the NASA/GISS general circulation model: the seasonal cycle for present-day conditions. J. Geophys. Res. $92,14,739-14,760$

Jouzel, J., Hoffmann, G., Koster, R.D., Masson, V., 2000. Water isotopes in precipitation; data/ model comparison for present-day and past climates. Quat. Sci. Rev. 19, 363-379.

Kendall, C., Coplen, T.B., 2001. Distribution of oxygen-18 and deuterium in river waters across the United States. Hydrol. Process. 15, 1363-1393.

Koch, P.L., 1998. Isotopic reconstruction of past continental environments. Annu. Rev. Earth Planet. Sci. 26, 573-613.

Koch, P.L., Tuross, N., Fogel, M.L., 1997. The effects of sample treatment and diagenesis on the isotopic integrity of carbonate in biogenic hydroxylapatite. J. Archaeol. Sci. 24, 417-429.

Kohn, M.J., 2004. Reviewed comment: tooth enamel mineralization in ungulates: implications for recovering a primary isotopic time-series, by Passey, B. H. and Cerling, T. E. (2002). Geochim. Cosmochim. Acta 68, 403-405.
Kohn, M.J., Cerling, T.E., 2002. Stable isotope compositions of biological apatite. In: Kohn, M.J., Rakovan, J., Hughes, J. (Eds.), Phosphates: Geochemical, Geobiological, and Materials Importance. Reviews in Mineralogy and Geochemistry, vol. 48. Mineralogical Society of America, Washington, D. C., pp. 455-488.

Kohn, M.J., Welker, J.M., 2005. On the temperature correlation of $\mathrm{d}^{18} \mathrm{O}$ in modern precipitation. Earth Planet. Sci. Lett. 231, 87-96.

Kohn, M.J., Miselis, J.L., Fremd, T.J., 2002. Oxygen isotope evidense for progressive uplift of the Cascade Range. Earth Planet. Sci. Lett. 204, 151-165.

Kohn, M.J., Schoeninger, M.J., Valley, J.W., 1998. Variability in herbivore tooth oxygen isotope compositions: reflections of seasonality or developmental physiology? Chem. Geol. 152, 97-112.

Krijgsman, W., Tauxe, L., 2004. Shallow bias in Mediterranean paleomagnetic directions caused by inclination error. Earth Planet. Sci. Lett. 222, 685-695.

Laskar, J., Robutel, P., Joutel, F., Gastineau, M., Correia, A.C.M., Levrard, B., 2004. A long term numerical solution for the insolation quantities of the Earth. Astron. Astrophys. $428,261-285$.

Lear, C.H., Elderfield, H., Wilson, P.A., 2000. Cenozoic deep-sea temperatures and global ice volumes from $\mathrm{Mg} / \mathrm{Ca}$ in benthic foraminiferal calcite. Science 287, 269-272.

Lee-Thorp, J.A., van der Merwe, N.J., 1987. Carbon isotope analysis of fossil bone apatite. S. Afr. J. Sci. 83, 712-715

Levin, N.E., Cerling, T.E., Passey, B.H., Harris, J.M., Ehleringer, J.R., 2006. A stable isotope aridity index for terrestrial environments. Proc. Natl. Acad. Sci. 103, 11201-11205.

Lionello, P., Malanotte-Rizzoli, P., Boscolo, R., Alpert, P., Artale, V., Li, L., Luterbacher, J., May, W., Trigo, R., Simplis, M.T., Ulbrich, U., Xoplaki, E., 2006. The Mediterranean climate: an overview of the main characteristics and issues. In: Lionello, P., Malanotte-Rizzoli, P., Boscolo, R. (Eds.), Mediterranean Climate Variability. Elsevier, Amsterdam, pp. 1-26.

Longinelli, A., 1984. Oxygen isotopes in mammal bone phosphate: a new tool for paleohydrological and paleoclimatological research? Geochim. Cosmochim. Acta 48, 385-390.

Luz, B., Kolodny, Y., Horowitz, M., 1984. Fractionation of oxygen isotopes between mammalian bone-phosphate and environmental drinking water. Geochim. Cosmochim. Acta 48, 1689-1693.

Montoya, P., Morales, J., Robles, F., Abella, J., Benavent, J.V., Marín, M., Ruiz, D., Sánchez, F.J. 2006. Las nuevas excavaciones (1995-2006) en el yacimiento del Mioceno final de Venta del Moro, Valencia. Estudios Geol. 62, 313-326.

Mosbrugger, V., Utescher, T., Dilcher, D., 2005. Cenozoic continental climatic evolution of Central Europe. Proc. Natl. Acad. Sci. 102, 14,964-14,969.

Nelson, S.V., 2005. Paleoseasonality inferred from equid teeth and intra-tooth isotopic variability. Palaeogeogr. Palaeoclimatol. Palaeoecol. 222, 122-144.

Nowak, R., 1999. Walker's Mammals of the World. Johns Hopkins University Press, Baltimore.

Opdyke, N., Mein, P., Moissenet, E., Pérez-Gonzalés, A., Lindsay, E., Petko, M., 1990. The magnetic stratigraphy of the Late Miocene sediments of the Cabriel Basin, Spain. In: Lindsay, E.H., Fahlbusch, V., Mein, P. (Eds.), European Neogene Mammal Chronology. Plenum, New York, pp. 507-514.

Pagani, M., Arthur, M.A., Freeman, K.H., 1999. Miocene evolution of atmospheric carbon dioxide. Paleoceanography 14, 273-292.

Passey, B.H., Cerling, T.E., 2002. Tooth enamel mineralization in ungulates: implications for recovering a primary isotopic time-series. Geochim. Cosmochim. Acta 18, 3225-3234.

Passey, B.H., Perkins, M.E., Voorhies, M.R., Cerling, T.E., Harris, J.M., Tucker, S.T., 2002. Timing of $\mathrm{C}_{4}$ biomass expansion and environmental change in the Great Plains: an isotopic record from fossil horses. J. Geol. 110, 123-140.

Pesquero, M.D., Alberdi, M.T., Alcalá, L., 2006. New species of Hipparion from La Roma 2 (Late Vallesian, Teruel, Spain): a study of the morphological and biometric variability of Hipparion primigenium. J. Paleontol. 80, 343-356.

Quade, J., Cerling, T., 1995. Expansion of C4 grasslands in the Late Miocene of Northern Pakistan - evidence from stable isotopes in paleosols. Palaeogeogr. Palaeoclimatol. Palaeoecol. 115, 91-116.

Quade, J., Solounias, N., Cerling, T.E., 1994. Stable isotopic evidence from paleosol carbonates and fossil teeth in Greece for $\mathrm{C} 3$ forest or woodlands over the past 11 Ma. Palaeogeogr. Palaeoclim. Palaeoecol. 108, 41-53.

Raymo, M.E., Grant, B., Horowitz, M., Rau, H., 1996. Mid-Pliocene warmth: stronger greenhouse and stronger conveyor. Mar. Micropaleontol. 27, 313-326.

Rink, W.J., Schwarz, H.P., 1995. Tests for diagenesis in tooth enamel: ESR dating signals and carbonate contents. J. Archaeol. Sci. 22, 251-255.

Rozanski, K., Araguas-Araguas, L., Gonfiantini, R., 1992. Relation between long-term trends of oxygen-18 isotope composition of precipitation and climate. Science 258 , 981-985.

Rozanski, K., Arguas-Araguas, L., Gonfiantini, R., 1993. Isotopic patterns in modern global precipitation. In: Swart, P. (Ed.), Climate Change in Continental Isotope Records. American Geophysical Union, Washington, pp. 1-36.

Ruddiman, W.F., Kutzbach, J.E., 1989. Forcing of Late Cenozoic Northern Hemisphere climate by plateau uplift in southern Asia and the America west. J. Geophys. Res. 94 18409-18427.

Sanchez Chillón, B.S., 1993. Aplicacion de tecnicas isotopicas $\left({ }^{18} \mathrm{O} /{ }^{16} \mathrm{O}\right)$ en la reconstruccion paleoclimatica de la Cuenca de Guadix-Baza durante el Plioceno Superior-Pleistocene Medio. Ph.D. thesis, Universidad Complutense, Madrid.

Sánchez-Chillón, B.S., Alberdi, M.T., Leone, G., Bonadonna, F.P., Stenni, B., Longinelli, A., 1994. Oxygen isotopic composition of fossil equid tooth and bone phosphate; an archive of difficult interpretation. Palaeogeogr. Palaeoclimatol. Palaeoecol. 107, 317-328.

Sharp, Z., Cerling, T., 1998. Fossil isotope records of seasonal climate and ecology: straight from the horse's mouth. Geology 26, 219-222.

Siegenthaler, U., Oeschger, H., 1980. Correlation of ${ }^{18} \mathrm{O}$ in precipitation with temperature and altitude. Nature 285, 314-317. 
Simón Gomez, J.L., 1983. Tectónica y neotectónica del sistema de fosas de Teruel. Teruel 69, 21-97.

Simón Gomez, J.L., 1991. Tectónica alpina y neotectónica del sistema de fosas de Teruel. In: Gutiérrez, M., Melendez, A. (Eds.), Introducción a al geologia de la provincia de Teruel. Instituto de Estudios Turlonese, Teruel, pp. 95-104.

Sinusia, C., Pueyo, E.L., Azanza, B., Pocovi, A., 2004. Datación magnetoestratigráfica de yacimiento paleontológico de Puebla de Valverde (Teruel). VI Congreso Geológico de España, 2004, Zaragoza.

Smith, B.N., Epstein, S., 1971. Two categories of. ${ }^{13} \mathrm{C} /{ }^{12} \mathrm{C}$ ratios for higher plants. Plant Physiol. 47, 380-384.

Sondaar, P.Y., 1961. Les Hipparions de l'Aragon meridional. Estud. Geol. 17, 209-305.

Tipple, B.J., Pagani, M., 2007. The early origins of terrestrial $C_{4}$ photosynthesis. Annu. Rev. Earth Planet. Sci. 35, 435-461.

Torsvik, T.H., Müller, R.D., Van der Voo, R., Steinberger, B., Gaina, C., 2008. Global plate motion frames: toward a unified model. Rev. Geophys. 46, RG3004. doi:10.1029/ 2007RG000227.

Tütken, T., Vennemann, T.W., Janz, H., Heizmann, E.P.J., 2006. Palaeoenvironment and palaeoclimate of the Middle Miocene lake in the Steinheim basin, SW Germany: reconstruction from $\mathrm{C}, \mathrm{O}$, and $\mathrm{Sr}$ isotopes of fossil remains. Palaeogeogr. Palaeoclimatol. Palaeoecol. 241, 457-491.

Utescher, T., Mosbrugger, V., Ashraf, A.R., 2000. Climate evolution in Northwest Germany over the last 25 million years. Palaios 15, 430-449.

van Dam, J.A., 2006. Geographic and temporal patterns in the late Neogene (12-3 Ma) aridification of Europe. The use of small mammals as paleoprecipitation proxies. Palaeogeogr. Palaeoclimatol. Palaeoecol. 238, 190-218.

van Dam, J.A., Weltje, G.J., 1999. Reconstruction of the late Miocene climate of Spain using rodent paleocommunity successions: an application of end-member modelling. Palaeogeogr. Palaeoclimatol. Palaeoecol. 151, 267-305.

van Dam, J.A., Alcalá, L., Alonso Zarza, A.M., Calvo, J.P., Garcés, M., Krijgsman, W., 2001. The Upper Miocene mammal record from the Teruel-Alfambra region (Spain): the
MN system and continental stage/age concepts discussed. J. Vertebr. Paleontol. 21, 367-385.

van Dam, J.A., Abdul Aziz, H., Álvarez Sierra, M.A., Hilgen, F.J., van den Hoek Ostende, L.W., Lourens, L., Mein, P., van der Meulen, A.J., Pelaez-Campomanes, P., 2006. Long-period astronomical forcing of mammal turnover. Nature 443, 687-691.

Van der Merwe, N.J., Medina, E., 1991. The canopy effect, carbon isotopic and foodwebs in Amazonia. J. Archaeol. Sci. 18, 249-259.

van der Meulen, A.J., Daams, R., 1992. Evolution of Early-Middle Miocene rodent faunas in relation to long-term palaeoenvironmental changes. Palaeogeogr. Palaeoclimatol. Palaeoecol. 93, 227-253.

Vallejos, A., Pulido-Bosch, A., Martin-Rosales, W., Calvache, L., 1997. Contribution of environmental isotopes to the understanding of complex hydrologic systems. A case study: Sierra de Gador, SE Spain. Earth Surf. Process. Landf. 22, 1157-1168.

Walcroft, A.S., Whitehead, D., Kelliher, F.M., Arneth, A., Silvester, W.B., 2002. The effects of long-term, partial shading on growth and photosynthesis in Pinus radiata D. Don trees. For. Ecol. Manag. 163, 151-163.

Wang, Y., Cerling, T.E., MacFadden, B.J., 1994. Fossil horses and carbon isotopes: new evidence for Cenozoic dietary, habitat, and ecosystem changes in North America. Palaeogeogr. Palaeoclimatol. Palaeoecol. 107, 269-279.

Wolfe, J.A., 1993. A method of obtaining climatic parameters from leaf assemblages. U.S. Geol. Surv. Bull. 2040, 1-71.

Yurtsever, Y., Gat, J.R., 1981. Atmospheric waters. In: Gat, J.R., Gonfiantini, R. (Eds.), Stable Isotope Hydrology-Deuterium and Oxygen-18 in the Water Cycle. IAEA, pp. 103-142.

Zachos, J., Pagani, M., Sloan, L., Thomas, E., Billups, K., 2001. Trends, rhythms, and aberrations in global climate 65 Ma to present. Science 292, 686-693.

Zazzo, A., Bocherens, H., Brunet, M., Beauvilain, A., Billiou, D., Mackaye, H.T., Vignaud, P., Mariotti, A., 2000. Herbivore paleodiet and paleoenvironmental changes in Chad during the Pliocene using stable isotope ratios of tooth enamel carbonate. Paleobiology 26, 294-309. 